\title{
Visual Analysis of Large Graphs: State-of-the-Art and Future Research Challenges
}

\author{
T. von Landesberger ${ }^{1,2}$, A. Kuijper ${ }^{1,2,3}$, T. Schreck ${ }^{1}$, J. Kohlhammer ${ }^{2}$, J.J. van Wijk ${ }^{4}$, J.-D. Fekete ${ }^{5}$ and D.W. Fellner ${ }^{1,2,3}$

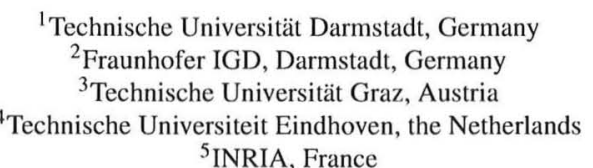 \\ \{tatiana.von_landesberger@gris.informatik.tu-darmstadt.de, arjan.kuijper@igd.fraunhofer.de, tobias.schreck@gris.tu-darmstadt.de, \\ joern.kohlhammer@igd.fraunhofer.de,vanwijk@win.tue.nl, Jean-daniel.fekete@inria.fr, d.fellner@igd.fraunhofer.de\}
}

\begin{abstract}
The analysis of large graphs plays a prominent role in various fields of research and is relevant in many important application areas. Effective visual analysis of graphs requires appropriate visual presentations in combination with respective user interaction facilities and algorithmic graph analysis methods. How to design appropriate graph analysis systems depends on many factors, including the type of graph describing the data, the analytical task at hand and the applicability of graph analysis methods. The most recent surveys of graph visualization and navigation techniques cover techniques that had been introduced until 2000 or concentrate only on graph layouts published until 2002. Recently, new techniques have been developed covering a broader range of graph types, such as timevarying graphs. Also, in accordance with ever growing amounts of graph-structured data becoming available, the inclusion of algorithmic graph analysis and interaction techniques becomes increasingly important. In this State-of-the-Art Report, we survey available techniques for the visual analysis of large graphs. Our review first considers graph visualization techniques according to the type of graphs supported. The visualization techniques form the basis for the presentation of interaction approaches suitable for visual graph exploration. As an important component of visual graph analysis, we discuss various graph algorithmic aspects useful for the different stages of the visual graph analysis process. We also present main open research challenges in this field.
\end{abstract}

Keywords: visual graph analysis, graph visualization, graph interaction, visual analytics

ACM CCS: Data Structures [E.1]: Graphs and Networks; Trees; Mathematics of Computing [G.2.2]: Discrete Mathematics; Graph Theory [H.4]: Information Systems: Applications; Information Systems [H.5.2]: Interfaces and Presentation; User Interfaces.

\section{Introduction}

The analysis of graphs is important in many application areas including finance, biology, sociology, transportation and software engineering. It includes a variety of different tasks. The main aspects relate to the understanding of global and local structure of the graph, the connections between entities, the clusters of highly connected entities, etc. Such high-level tasks often consist of a series of low-level tasks [LPS*06], in particular when dealing with large and complex graphs.
The analysis of graphs is often supported by their visual presentations. In this respect, graph visualization research concentrates on the development of effective graph layouts and visual mappings. The visualization of large graphs is accompanied by effective interaction techniques, in particular, in cases when the whole graph is too complex or large to be visualized in one static view. The interaction alone may not be sufficient to accomplish certain analytical tasks. Therefore, algorithmic support-such as machine learning, or graph analysis algorithms-needs to be supported in 


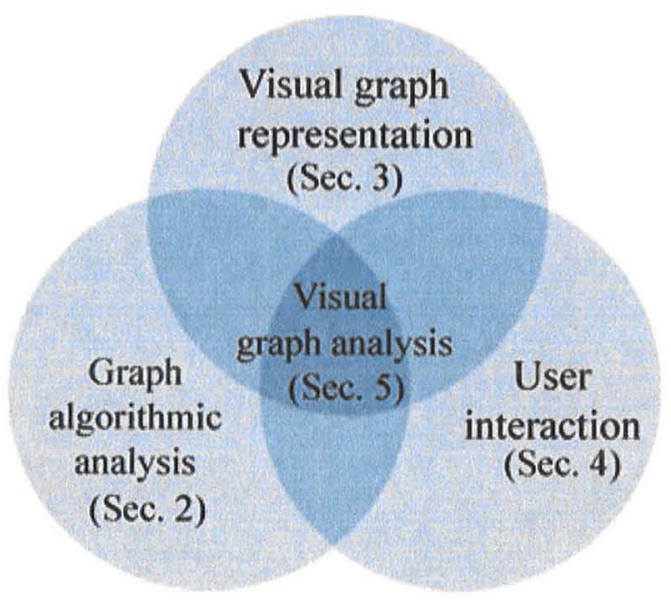

Figure 1: The main components of visual graph analysis considered in this report.

interactive visualization systems. Such integrated visual analysis of large data sets is the main focus of the research field called Visual Analytics, which evolved from Information Visualization and Scientific Visualization [KMS*08]. It has effectively started to grow after the publication of the seminal book by Thomas and Cook in 2005 [TC05]. Therein, Visual Analytics is defined as the science of analytical reasoning facilitated by interactive visual interfaces. Recently, Visual Analytics has been a major driving force for the research and development of interactive visualization techniques for large amounts of data including graphs.

Our motivation for this report is twofold. First, we recognize that by now most recent graph visualization survey [HMM00, DPS02] date back several years. Therefore, we aim to provide an update by adding more recent publications to the body of work presented in these surveys. Secondly, we aim to take a Visual Analytics perspective on the field of visual graph analysis by explicitly considering in a unified way the aspects of visual representation, user interaction, and algorithmic analysis (Figure 1). These three elements form the basis for effective visual graph analysis systems and are closely interrelated. For example, algorithmic graph analysis may be applied as a pre-processing step before a specific graph layout is determined for visual representation. Interactive direct object manipulation approaches are often useful for exploring large and complex graphs visually. Also, by means of user interaction, further graph analytic processing steps, or updates to the presented views, can be requested. The algorithmic analysis thereby helps to reveal interesting aspects of the data. The user involvement in this analytic process can vary from an automatic analysis, where the calculation is done without user involvement, over a user-driven analysis, where the user triggers the algorithmic processing of the data, up to a user-steered process where the user has

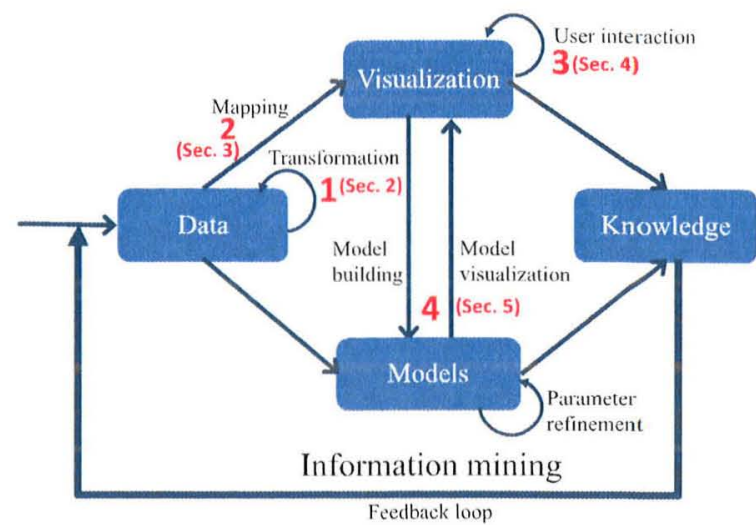

Figure 2: The Visual Analytics process by Keim et al. [KAF*08] with the four key steps: (1) data pre-processing, (2) mapping/layout, (3) visual user interaction and (4) modelbased analysis. $c 2008$ Springer-Verlag, Berlin, Heidelberg.

full control over the analytical process including setting the algorithm parameters.

In this state-of-the-art report, we provide a systematic overview of the main approaches in each of the three aspects of visual analysis of graphs. We therefore develop a classification of techniques according to these aspects. Within each category, we refer to exemplary papers, while focusing on new developments in the visual graph analysis area. We discuss the respective techniques to offer the reader the possibility to concentrate on methodological aspects of visual analysis of graphs applicable across various domains. Owing to the broad scope of the paper, we present the main features (strengths and weaknesses) of the techniques as far as they were discussed by the authors of the papers or were mentioned in evaluations.

The structure of the report reflects the steps of the visual analytics process introduced by Keim et al. [KAF*08] (Figure 2). Section 2 details on definitions and a classification of graphs by types and introduces main pre-processing methods for visual graph analysis. This section is the basis for a discussion of visual graph representations given in Section 3 . Sections 4 and 5 survey key approaches for interaction with and algorithmic analysis of graphs in visualization, respectively. Finally, Section 6 concludes and outlines future challenges in this research domain.

\section{Basic Graph Definition and Pre-processing Techniques}

In this section, we recall fundamental graph definitions as well as approaches for graph pre-processing useful for subsequent graph visualization. 


\subsection{Definitions}

Graphs are a prominent data structure within Visual Analytics and related research fields. Often, graphs are applied for describing relationships between entities. A graph refers to a set of vertices (nodes) and a set of edges (i.e. links) that connect pairs of vertices. It is a pair $G=(V, E) ; E \subseteq\left[V^{2}\right]$, where elements of $V$ are vertices and elements of $E$ are edges [Die05]. Furthermore, attributes can be attached to vertices and edges, for example to denote their type, size or some other application-related information.

Graphs are often classified into undirected and directed [HMM00]. For a directed graph (resp. undirected), the edge vertices $e=\left(v_{1}, v_{2}\right)$ are ordered (resp. unordered). A graph containing both directed and undirected edges is called mixed.

A path of length $s$ in $G$ is a sequence of connected vertices $\operatorname{path}_{G}\left(v_{1}, v_{s}\right)=v_{1}, v_{2}, \ldots, v_{s}$ where $v_{i} \in V$ and $\left(v_{i}, v_{i+1}\right) \in$ $E$. A cycle is a closed path with $a_{1}=a_{s}$. A tree is a connected undirected graph without cycles [Die05]. A connected graph can be transformed to a tree by removing edges causing cycles while keeping the graph connected. A Tree $T$ is called rooted when one vertex $r$ is distinguished as a so-called root node: $T=(V, E, r)$. Such trees are often treated as hierarchies, where the length of the path to the root denotes the level of a vertex in the hierarchy. We note that, formally, a hierarchy is a directed acyclic graph so, there can be several paths from a vertex to the root node. In this survey, we use the term hierarchy as synonym to 'rooted tree'.

In graph theory literature, a directed graph with weighted edges is also called a network. In information visualization, the term network is often used in a broader sense denoting a graph with attributes associated with vertices and edges.

An additional graph category are so-called compound graphs. A compound graph $C=(G, T)$ is defined as a graph $G=\left(V, E_{G}\right)$ and a rooted tree $T=\left(V, E_{T}, r\right)$ that share the same set of vertices, such as

$\forall e=\left(v_{1}, v_{2}\right) \in E_{G}, v_{1} \notin \operatorname{path}_{T}\left(r, v_{2}\right)$ and $v_{2} \notin \operatorname{path}_{T}\left(r, v_{1}\right)$.

Relationships between vertices are expressed by $T$ : vertices sharing a common parent in $T$ belong to the same 'group'. When two vertices sharing a common parent are connected in $G$, they share a generic relationship. Many other kinds of relationships can be expressed including hierarchic and cross group.

Compound graphs can be created by successive aggregation (or clustering) of graph vertices in a bottom-up approach. This operation usually involves creating new nodes as group/cluster parents. In this case, vertices (and implicitly, also edges) of the original graph are aggregated (i.e. added as children of the group parent), thereby creating constructed meta-nodes or super-nodes. The attributes of the meta-nodes can be calculated from the attributes of the merged nodes.

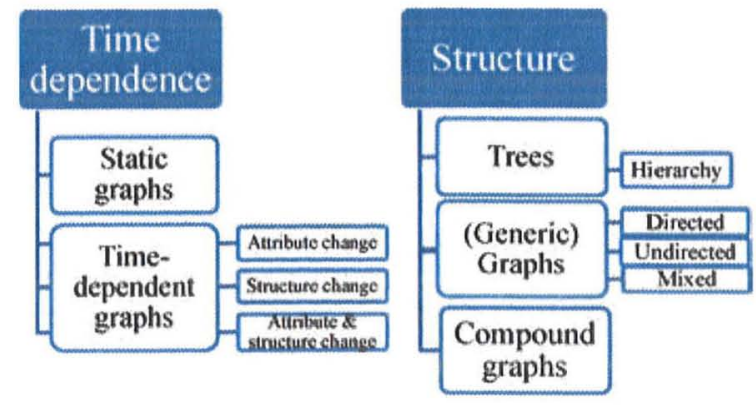

Figure 3: Classification of graphs according to their time dependence and graph structure.

Similarly, edges between meta-nodes are aggregated into meta-edges and their attributes can be calculated from the original edges. Compound graphs which are constructed in this way are also referred to as aggregated graphs. The list of operations that can be performed on such graphs is dependent on the particular application and graph type.

Graphs may also evolve over time, thereby forming $d y$ namic graphs (i.e. time-dependent graphs) in contrast to static graphs. Time-dependent changes may affect the attributes of nodes and edges, the graph structure or both Figure 3 summarizes the graph classification presented earlier.

Furthermore, graphs may be distinguished according to their topological properties. There exists a variety of literature on graph theory (e.g. [Die05]) which focuses on graph terminology, classification and algorithmic graph analysis. In the following, we mention only the most relevant terminology used later in this report. Basic graph properties include the number of nodes, graph density and connectivity. Properties are often taken into account (or are a pre-requisite) for certain visualization techniques. These properties influence the choice and effectiveness of the applied visualization methods. For example, the increasing number of nodes, higher graph density or both pose a scalability problem in visualization owing to limited display space and human perception capabilities.

The number of nodes (i.e. graph order) is often referred to as graph size $(|V|)$. Graph density is the number of edges relative to the maximum potential number of edges $D=\frac{2|E|}{|V|(|V|-1)}$. Sparse graphs have around $O(|V|)<|E| \ll$ $O\left(|V|^{2}\right)$ edges, whereas dense graphs show density values close to one. Graphs with the maximum number of edges are called complete graphs. A clique is a subset of a graph that is fully connected.

According to the graph size, graphs are often referred to, for example as small or large. The definition of large graphs is however not standardized. Often graphs with thousands, hundreds of thousands or millions of nodes are called large. However, not only the number of nodes determines the 
notion of a 'large' graph. Graph density and connectivity also play an important role for the notion of a 'large' graph. From the visualization point of view, 'large' graphs usually lead to cluttered displays. In algorithmic analysis, 'large' graphs refer to long computational times or memory footprint larger than the available RAM size. A discussion about the influence of graph size and density on visualization and construction of graphs for testing visualizations according to these parameters is provided in [Mel06].

Several special graph structures appear often in real-world cases, and dedicated visualization methods have been developed for these [ACJM03, vHW08, JHGH08, MJW*09]. For example, social networks usually exhibit a structure called small world network: the typical distance between two nodes grows proportionally to $\log |V|$. Scale-free networks, for example protein networks or certain types of social networks have a degree distribution that follow approximately the power law. Bipartite graphs are graphs whose nodes form two disjoint sets: $V_{1}, V_{2}$ with $V_{1} \cup V_{2}=V$ and $V_{1} \cap V_{2}=$ $\emptyset$, such that: $\forall e=\left(v_{1}, v_{2}\right) \in E, v_{1} \in V_{1}$ and $v_{2} \in V_{2}$.

\subsection{Algorithmic graph pre-processing}

In graph visualization, algorithmic graph pre-processing often includes graph simplification to reduce the size, while maintaining the main graph structure. Also pre-processing of graph properties can be used for graph visualization (in algorithms for positioning of nodes and edges) or highlighting of interesting parts of the graph. The modified graph is used then for an easier visual inspection as large and complex graphs are difficult to understand even using advanced node and edge positioning algorithms (layouts). Such pre-processing steps can usually be performed automatically without user interaction. There are two main approaches to graph reduction: graph filtering and graph aggregation.

\subsection{Graph filtering}

There are two types of filtering: stochastic and deterministic. Stochastic filtering is mainly based on random selection of nodes and edges from the original graph. These methods are compared in [LF(6)]. Deterministic filtering uses, as its name suggests, a deterministic algorithm for the selection of the nodes/edges to be removed. This filtering can be based on node/edge attributes, on topologic values such as betweenness centrality or other graph properties. For example, filtering based on edge-betweenness-centrality can be used for removal of less important edges while keeping the underlying structure (connectedness and other features such as cliques) of the graph [JHGH08] (Figure 4).

\subsection{Graph aggregation}

In this approach, nodes and edges are merged to single nodes and edges, thereby reducing the size of the graph and revealing relationships between groups of nodes. Graph ag-

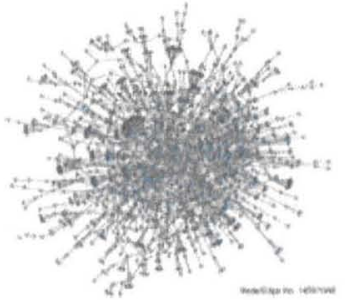

(a) Original graph

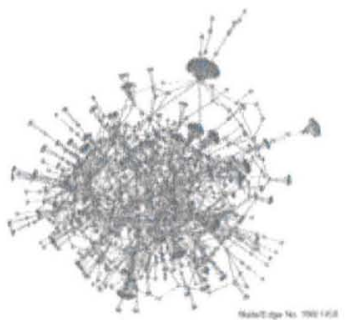

(c) Geodesic clustering

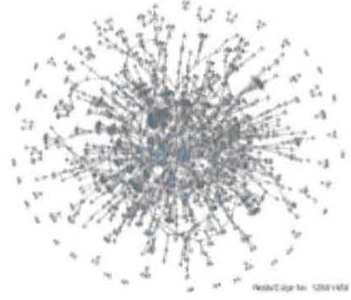

(b) Stochastic edge sampling

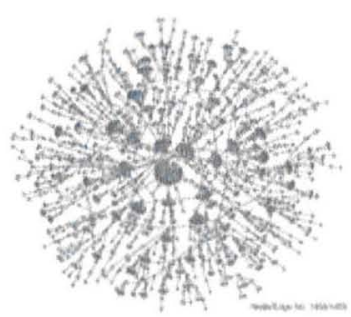

(d) Structure-based filtering
Figure 4: Example of various graph reduction techniques. The graphs are visualized using the GEM layout [JHGH08]. (c) 2008 IEEE.

gregation can be repeated multiple times, creating a hierarchical graph, which is a special kind of compound graph. There are various ways of aggregating a graph, including using predefined node hierarchies, or aggregation according to node attributes, or according to the node clusters [EDG*08, BDL*10], to name a few. Figure 5 (top) shows an example aggregation schema with several aggregation levels.
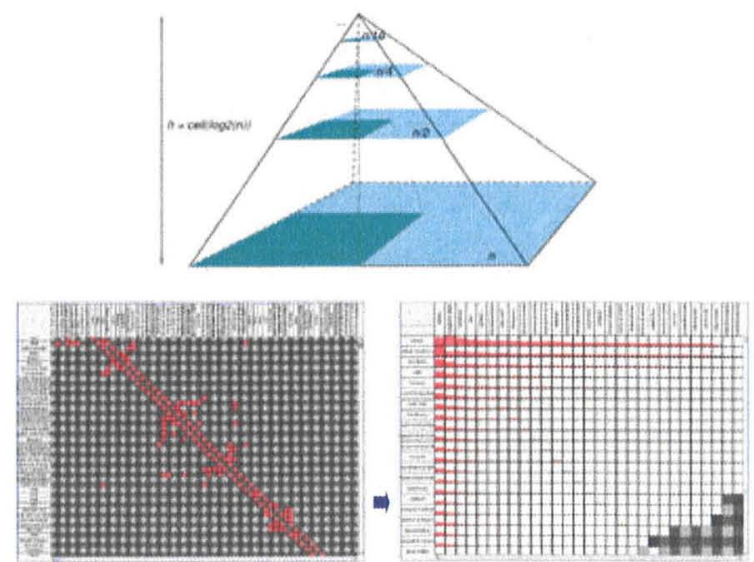

Figure 5: Graph aggregation for multiscale graph visualization [EDG*08]. c 2008 IEEE. Top: Graph aggregation schema showing several levels of aggregation. Darker rectangles show the corresponding data areas in the aggregation. Bottom: Example of graph aggregation using a matrix visualization. 
The highlighted rectangle shows the corresponding data in each aggregation level. Figure 5 (bottom) shows the original and aggregated data in a matrix visualization.

\section{Visual Representations of Graphs}

Visualization is one of the main means of exploratory graph analysis. It includes the development of appropriate types of visual representations (e.g. matrix or node-link diagrams), efficient placement of graph elements on the screen and efficient visual attribute mappings (design of graph elements for improved readability of the drawing).

In computer-created graph visualization, several so-called aesthetic criteria are taken into consideration. They are usually implemented as objective functions to optimize in layout algorithms. The standard criteria include minimizing the number of crossings, minimizing the total drawing area, maximizing symmetries and many more related to particular types of graphs and edge drawing styles [Pur97, DBETT99, BBD09]. Recently, Beck et al. [BRSG07] extended previous works to focus on both static and dynamic graphs irrespective of their graphic representations (including also matrix representations in addition to node-link diagrams). They consider three groups of criteria: general, dynamic and aesthetic scalability.

- The general criteria include reduction of visual clutter, reduction of spatial misunderstanding resulting from spatial closeness, maximization of spatial matching of items for following paths and maximization of space efficiency.

- For dynamic graphs, the following criteria are desired: maximization of display stability between time points, reduction of cognitive load when analysing time dynamics, minimization of temporal aliases mainly owing to positioning of different nodes in the same place in two time periods.

- Aesthetic scalability criteria refer to graph readability for larger graphs, that is, scalability in number of vertices (i.e. increasing graph order), scalability in number of edges (i.e. increasing graph density), and scalability in number of graphs, in particular with increasing number of time steps for which graph data is given.

All these criteria are important but they cannot be simultaneously optimized and are not sufficient to design a good layout which is usually data and task dependent. Therefore, exploratory graph visualization requires more than one layout algorithm to reveal the several perspectives on relationships between nodes.

In this section, we describe the main graph visualization techniques following the graph classification from Section 2. We introduce techniques for static and time-varying graphs. In each part, techniques for hierarchies, generic directed and undirected graphs and compound graphs are presented. We discuss different ways of visual graph representations and designs of graph drawings.

\subsection{Visual representations of static graphs}

The visualization of static graphs has received much attention in the Information Visualization community. The section start with trees that are simpler than general graphs.

\subsubsection{Trees}

Techniques for displaying trees can be divided into three main groups: Space filling, node-link based and hybrid (Figure 6). There have been several studies comparing the different ways of visualizing trees [SCGM00, BN01, vHvW02, Kob04, AK07]. A very useful visual overview of tree visualization has been provided in the poster [JS10]. It is difficult to unify these results as they differ significantly. Recently, Ziemkiewicz and Kosara have shown that the effectiveness of the visualization technique depends not only on the task to be solved, but also on the formulation of the task assignment, that is if it reflects a containment or a level metaphor [ZK08].

Node-link techniques: These approaches use links between items to depict their relationship. Layout algorithms controlled by optimization criteria or the node positions. Many layout algorithms have been proposed to date in the Graph Drawing community. They include layered, radial or balloon layouts in two-dimensional (2D) [HMM00], Cone trees [RMC91] in 3D, point-based trees [SSH09], nature inspired Phyllotrees [NCA06] or hyperbolic layouts [LRP95,

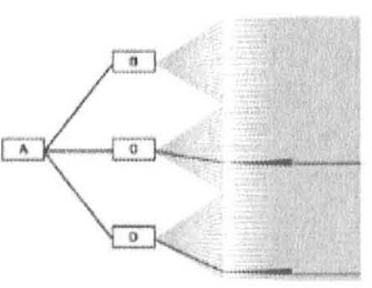

(a) Node-link diagram

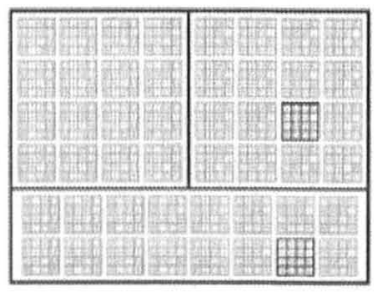

(b) Space-filling diagram

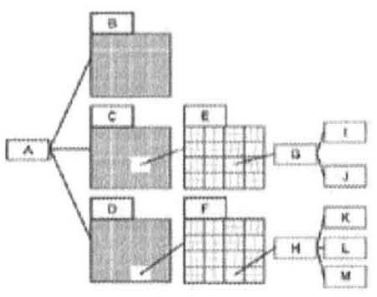

(c) Combined representation

Figure 6: Three types of hierarchy visualization techniques. (a) Node-Link, (b) space-filling and (c) hybrid. [ZMC05], c 2005 IEEE. 


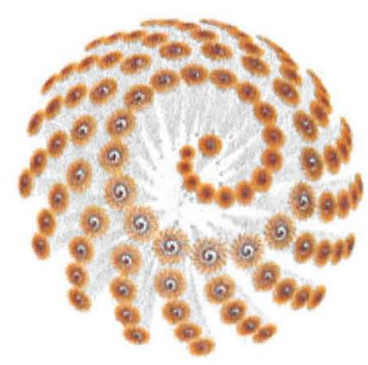

(a) Phyllotrees

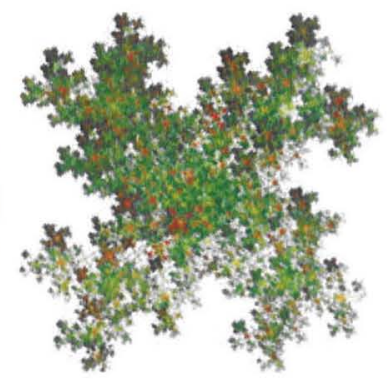

(b) Point-based tree
Figure 7: Examples of node-link tree visualizations. (a) Phyllotrees [NCA06], c 2006 IEEE. (b) Point-based tree [SSH09], c 2009 IEEE.

Mun97, AH98] (Figure 7). Most of these classic tree layout algorithms have a linear complexity in time and memory so the layout computation is scalable. However, the nodelink representation by design leaves significant background space empty and thereby may encounter scalability problems when applied to larger graphs. For the visualization of node attributes, specialized techniques for multidimensional data visualization such as glyphs, radial or parallel plots have been used.

Space filling techniques: These techniques try to use the full area of the display to present the hierarchy. Instead of employing links for representing node relationships, the spatial positions of nodes are employed, using either closeness or enclosure. They are mainly applied to visualization of hierarchic partitions of sets of data items, for instance files in a file system. Area size can be used to encode quantitative attributes of nodes, such as file size. In addition, colour and height can represent additional data attributes. In case more complex additional information needs to be displayed, specialized data presentations can be placed in the child nodes such as icons, parallel coordinate diagrams, etc. Space-filling techniques can be categorized by the placement strategy employed into enclosure, adjacency and crossing (Figure 8).

- Enclosures: These techniques recursively layout child nodes within the area of their parent nodes. The most prominent examples are Treemaps-rectangular shapes recursively subdividing rectangular display space according to the underlying hierarchy, introduced by Shneiderman[Shn92] (so-called slice-and-dice algorithm). Variants include Voronoi tessellations [BDL05] or bubble layouts [Bed01]. Other types, such as elliptic [OCNF09] or circular shapes have been proposed, but they do not lead to fully space filling visualizations.

The main advantage of enclosures is the very good usage of the available space, as the child nodes do not need extra space owing to the overlap with the parent nodes. The disadvantage is that the overlapping of the

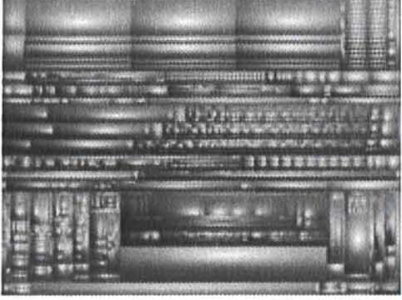

(a) Treemap

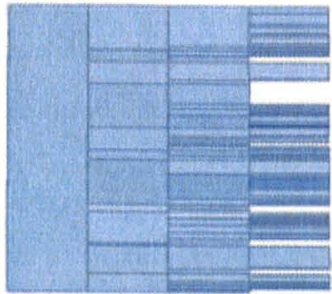

(b) Icicle plot

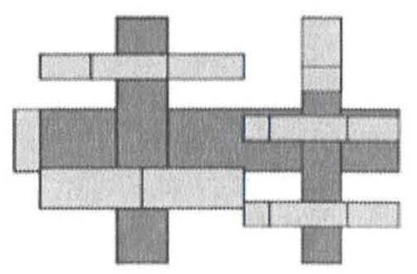

(c) BeamTree

Figure 8: Three types of space filling hierarchy visualization techniques. (a) Enclosure: Cushion Treemap [vWvdW99], (c 1999 IEEE. (b) Adjacency: Icicle plot [TS08], c 2008 IEEE. (c) Crossing: BeamTrees [vHvW02], c 2002 IEEE.

parent nodes may also lead to a more difficult distinction of the hierarchy structure by the user, as it is rather implicitly encoded. For Treemaps, several advanced layout techniques have been developed including ordered (i.e. pivot-based) [BSW02], squarified [BHvW99] and spiral [TS07] Treemap layouts. For example, squarified Treemaps aim at generating subrectangles of square-like aspect ratios, supporting easier comparison of sizes and presentation of additional diagrams or other elements within the rectangles. According to Tu and Shen [TS07], the slice-and-dice algorithm leads to high aspect ratios with good readability. Strip, pivot-based and spiral techniques have medium aspect ratios with medium readability. Squarified Treemaps have very good (low) aspect ratios but low readability. To better distinguish the hierarchical structure, cushion Treemaps [vWvdW99] apply shading of the shapes. Treemaps that reflect the geographic distribution of the hierarchical data were presented in [WD08].

- Adjacency: In contrast to Treemaps, adjacency-based techniques do not overlap the parent nodes by child nodes, but represent the node relationships by placing the child nodes next to their parent nodes. The placement can be in circular layers, such as in the SunBurst method [SZ00], or on linear layers, yielding so-called icicle plots. The advantage of this visualization is that the parent nodes are not overlapped by their child nodes and therefore, their attributes can be more easily displayed and analysed. However, this visualization is not as dense as squarified Treemaps. 
- Crossings: The crossing method places child nodes across the parent node, thereby only partially overlapping the parent. The Beamtree method [vHvW02] improves over the classic Treemaps when the hierarchical structure may be difficult to visually assess, while still being more space efficient than the adjacency techniques. The main drawback of this technique is that users are unfamiliar with this approach and that it is often less readable than other methods.

Hybrid approaches: These approaches combine nodelink diagrams with Treemaps: a part of the hierarchy is displayed in a Treemap and the rest as a node-link diagram (Figure $6 \mathrm{c}$ ). They present the data in a flexible space-efficient way while still clearly presenting the data structure and emphasizing the content. The most prominent representative are 'elastic hierarchies' [ZMC05]. In connection to interactive determination of the type of visual metaphor used for each part of the hierarchy, this technique allows for flexible analysis of the data using advantages of both representations.

\subsubsection{Directed and undirected graphs}

Techniques for displaying general graphs can be divided into three main groups: node-link based, matrix-based and hybrid (Figure 9). We discuss these in more detail later. In addition, there are specialized graph drawing techniques, which use new graph visualization techniques. Two main examples are: graph splatting and graph maps. The first one forms graphs as 2D scalar fields [vLdL03]. The second one visualizes graphs as maps [GHK10], where the relationships between nodes are represented as adjacency between neighbouring areas (nodes). Both approaches create an approximate representation of a graph.

A comparison of node-link and matrix techniques is presented by Ghoniem et al. [GFC04]. According to the study, the advantages of node-link diagrams are their intuitiveness, compactness and better suitability for path following tasks. They are more effective for smaller and sparse graphs. The

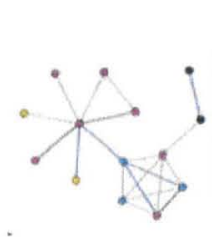

(a) Node-link diagram

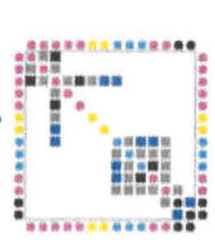

(b) Adjacency matrix diagram

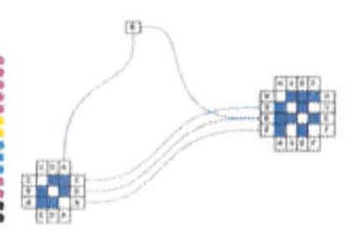

(c) Combination
Figure 9: Three types of general graph visualization techniques: a) Node-link diagram, b) adjacency matrix, c) hybrid. From [HFM07], c 2007 IEEE.
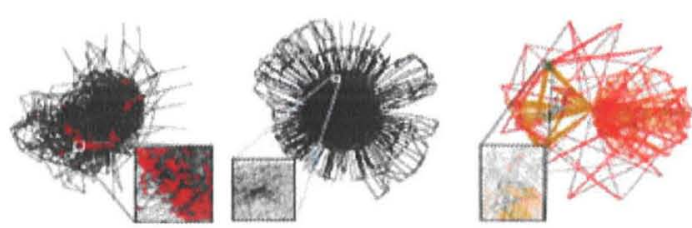

(a) Multi-level graph layouts

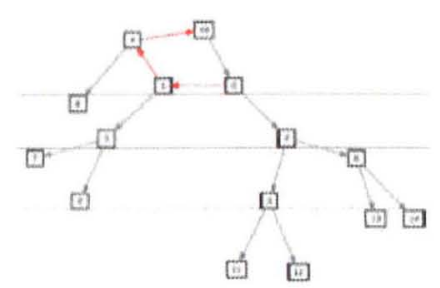

(b) Dig-Cola layout

Figure 10: Graph layout examples. (a) A comparison of multi-level graph layouts GRIP, FM ${ }^{3}$ and Topolayout [AAM07]. (c) 2007 IEEE. (b) Layered layout of cyclic directed graph [DK05]. c 2005 IEEE.

Matrix representation inherently does not have edge crossings and node overlapping problems, and is thereby suitable also for dense graphs. When using appropriate node ordering, they can easily reveal dense substructures in the graph. However, they also suffer from scalability in limited display spaces, especially for very large graphs. In visual graph analysis, graph layout and matrix ordering influence the effectiveness of these representations. These issues are therefore in the core of graph visualization research.

Node-link representations: The main challenge is the layout (i.e. the placement of the nodes) so that graph readability and certain notions of graph aesthetics are supported (Figure 10). Typical requirements include that the nodes do not overlap, the number of edge crossings is minimized, edge length is homogeneous and in general, graph substructures are easily recognizable. This problem is intensively studied in the Graph Drawing community. Given these aesthetic goals and constraints, the aim is to find algorithms that efficiently provide good solutions.

Note that a specific group of graphs are graphs with geographic reference, such as transportation graphs. In this case, the nodes and possibly also edges of the graph have an inherent geographic location, which needs to be taken into consideration in their graphic presentation. Therefore, a specific graph layout algorithm is not needed for determining the position of each node on the screen. However, the fixed node position may exacerbate graph readability problems, such as crossings and long edges. Visualization of geographic data is a special research field, which we do not address here in detail. 
When no position is inherently associated with vertices, a graph layout algorithm is required. The graph layout research field is very large, and an extensive survey of proposed techniques is beyond the scope of this report. The latest survey from Herman et al. dates from 2000 [HMM00] and several new algorithms have appeared since then. The related work part in [AAM07, MM08] as well as the comparison in [HJ07] nicely summarize many current techniques. In our report, we classify the techniques according to the type of node placement.

- Force-based layouts: These techniques are based on a simulation of mechanical laws by assigning repulsive forces between nodes and attraction forces between endpoints of edges. Several forces have been described in the literature to achieve different properties of the layout. The seminal work of Eades [Ead84] uses an electric force between charged particles to model node repulsion and spring forces between the link endpoints to model edge attraction. Fruchterman and Reingold [FR91] have then improved the distribution of nodes by adaptation of the force models and Noack has further improved it with a more flexible set of force functions to achieve either a good space density or a good clustering of nodes [Noa03]. Kamada and Kawai [KK89] try to layout nodes such as the Euclidean distance between the nodes is proportional to the graph-theoretical distance. This family of layouts, however, does not scale well to graphs of thousands of nodes or more, due to their complexity. Therefore, improvements have been proposed. For instance, faster calculation of forces using an efficient GPU implementation [GHGH09], or using heuristics [FLM95].

- Constraint-based layouts: This family of layouts extends the force-directed approach with constraints on node position. These constraints include horizontal and vertical alignment of nodes, nonoverlapping nodes, edge direction, or closeness of grouped nodes [DMW09]. An example are orthogonal layouts, where the edges are only composed of straight vertical and horizontal lines. These layouts can be supported also by user interaction (see also Section 4). Example works from this category include [DMS*08, DMW09, DMW09]. This family of layouts greatly improves the power of expression at the cost of slightly longer execution time.

- Multiscale approaches: These techniques rely on a hierarchical decomposition of a graph into simpler nested sub-graphs. They first layout the coarser graph and then include more nodes level by level. Exemplary works include [GK01, KCH02, HJ05, FT07, MM08] (Figure 10a). These methods are typically much faster than traditional force-directed methods. They can be differentiated according to the technique used for creating the node hierarchy, and the layout of the resulting layers. For example, [MM08] employs node clustering and subsequent positioning of the nodes along space filling curves.

- Layered layouts: These approaches, also called 'hierarchic layouts', place nodes of the graph on parallel horizontal layers [GKNV93]. They are mainly used for directed graphs and are based on the Sugiyama approach [STT81]. It works in four phases: (1) cycle removal, (2) assignment of nodes to layers, (3) reduction of edge crossings and (4) assignment of coordinates to nodes. Improvements to these layouts, specifically for cyclic graphs, position all nodes of a cycle within one level; examples include the Dig-Cola layout [DK05] and Cyclic Leveling [BBBL09] (Figure 10b). This algorithm and its variants are quite fast in practice and standard implementations such as [GKNV93] can easily layout several thousands of nodes in seconds.

- Non-standard layouts: Other approaches exist that combine the previous techniques or use completely alternative approaches to graph layouts. Projection of a node layout from high-dimensional to 2D space has been proposed in [HK02]; although it is very fast in practice, the quality of the layout is very sensitive to the structure of the graph. For example, it is very effective for meshes and not effective at all for trees. LGL [ADWM04] first simplifies the graph by computing a spanning-tree; it then computes the layout iteratively in depth order using a force-directed layout. LGL is able to scale to very large graphs (billions of vertices) thanks to the initial decomposition. It is very effective for quasi-trees but has not been thoroughly studied for other kinds of graphs; its results are very sensitive to the spanning-tree computation: choosing different spanning trees will results in quite different layouts for the same graph. The ISOM method [Mey98] applies the Self-Organizing Map algorithm [Koh01] for finding a suitable graph layout. As an alternative to costly layout computation, a graph layout visualization based on the semantics of the graph (on node labels) was presented in [SA06]. Semantically identical nodes (e.g. with the same labels) can be placed in boxes using standard layout algorithms (e.g. forcedirected) (Figure 11) or in layers using their importance for assigning the position within layers [GOB*10]. Furthermore, attributes or properties associated with graph vertices can be used directly to specify the position of these vertices, as with scatterplots [SA06, BCD*10]: the layout computation is then straightforward and very fast.

Comparison of graph layouts: A recent comparison of the readability of graph layouts using eye-tracking [Hua07, PSD09] has shown that force directed layouts outperform orthogonal and layered layouts on various user tasks. Another comparison of advantages and disadvantages of numerous current layouts was published by Hachul and Jünger [HJ07]. They compare the graph drawing outputs according to various criteria finding that the HDE layout [HK02] is 


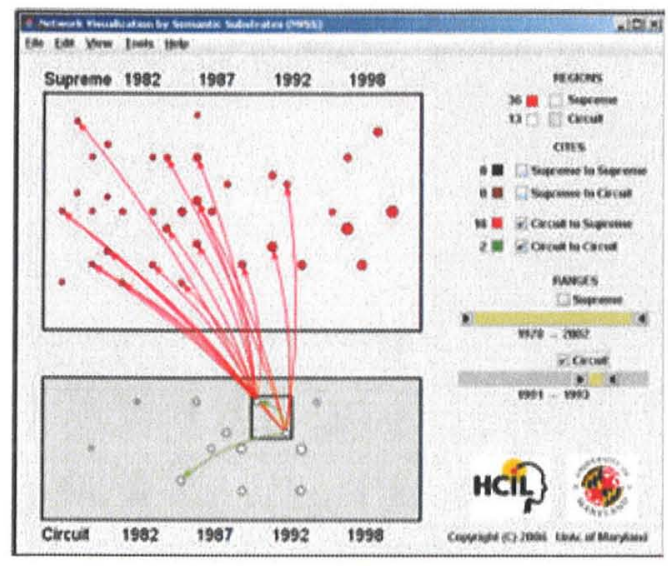

Figure 11: Graph visualization using data semantics [SA06]. c) 2006 IEEE.

very fast but frequently produces layouts with many overlapping edges. In contrast, $\mathrm{FM}^{3}$ [HJ05] creates pleasing layouts in reasonable time. Both algorithms together with GRIP [GK01] scale well with graph size. A comparison of user-produced versus automatically generated layouts [vHR08, DLF*09] found also that the results of physicsbased algorithms, such as force-directed layouts, were preferred by the users.

Design of graph drawing: The above-mentioned techniques cover graph layout. In addition to specific layouts, occlusion and readability of the display can be improved by edge-bundling [Hol06, CZQ*08, TE10, LBA10] (Figure 12) and the removal of node overlap [GH09, IAG*09]. Drawing of node-link diagrams also includes a suitable design of edge and node drawing primitives. For directed graphs, the representation of edge directions is of importance. There are multiple design possibilities including usage of arrows, colour transitions (from colour A to colour B), thickness

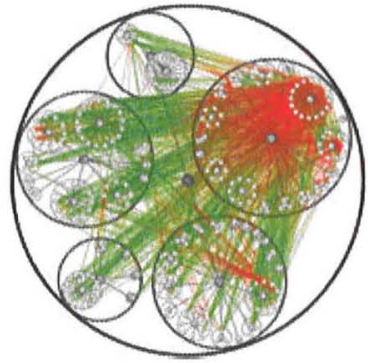

(a) Original graph

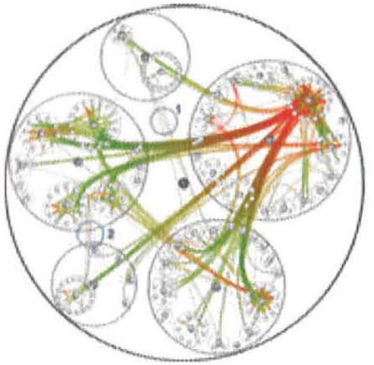

(b) Edge bundling
Figure 12: The use of edge bundling for improving graph readability. (a) Ariginal graph and (b) graph with edge bundling. [Hol06], c 2006 IEEE. transitions (from thick to narrow), curves and animated textures [TK08, HvW09, BBG*09]. These options may also be combined. A comparison of graph drawing different ways to represent edges was presented in [HvW09]. It shows that arrows, although popular and widely used, do not perform as well as colour and thickness transitions. Graph nodes and edges often have associated attributes that are included in the analysis. This study did not concentrate on attributed edges. For such edge attributes, in particular edge weight, colouring of edges or edge thickness can be employed. For the visualization of node attributes, a visualization of multivariate data items (e.g. glyphs or radial plots) is employed. Various possibilities of graph designs can be found in [Kre09].

Visualization of multiple graph connected components: For the visualization of multiple components, first a layout for each individual connected component is calculated and then a specific placement of these components on the screen is performed. The most widely used placement method is called packing. It lays out the components so that they do not overlap and are space efficient. Dogrusoz [Dog02] compares several 2D packing algorithms for graphs which use representation of graphs by their bounding rectangles. They include strip packing, tiling and alternate-bisection. The polyomino algorithm of Freivalds et al. [FDK02] uses a special representation of the graph objects, which substantially reduces the unused display space in comparison to rectangular shapes. Goehlsdorf et al. [GKS07] introduce new quality measures to evaluate a $2 \mathrm{D}$ placement which yields more compact layouts than the previously mentioned approaches.

Matrix representation: These techniques visualize the adjacency matrix of a given graph, where edge attributes are encoded in the matrix cells. They can display both directed and undirected graphs, where the latter leads to a symmetric matrix. The advantage of this representation with respect to the node-link representation is the non-overlapping display of graph edges, and the readability of the graph especially for larger and denser graphs. The disadvantage is an increased difficulty for users to follow paths, and a possible unfamiliarity of matrices to the users. In a matrix visualization, the ordering of rows/columns plays an important role: similar to layout for the node-link representation. Different strategies to order the matrix can be employed (Figure 13). Prespicuous reordering can reveal clusters in the graph and other patterns. For a discussion of these, we refer to [MML07, DPS02, $\mathrm{HF} 06, \mathrm{EDG}^{*} 08$ ]. Although matrices are suitable for larger graphs, they also suffer from scalability issues as they use linear order of nodes along the matrix rows/columns. Therefore, interaction techniques and aggregated displays have been proposed [vH03, AvH04, HF06, EDG*08, vHSD09] (see also Sections 4 and 5).

Combination of matrix and node-link approach: Techniques using a combination of the two previous approaches aim at overcoming their limitations by focusing on their strengths. Three main approaches exist (Figure 14). 


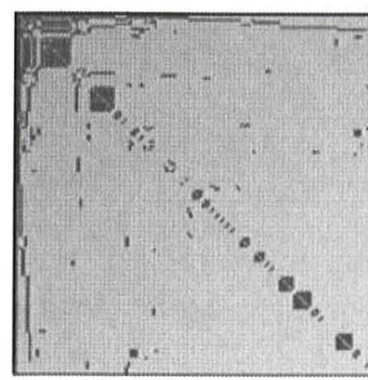

(a) HDE matrix ordering

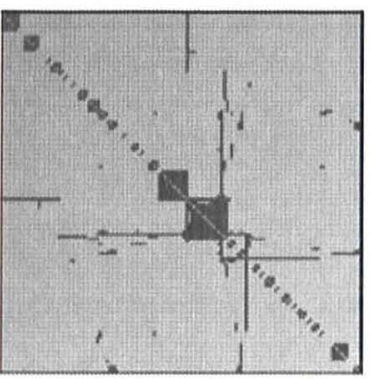

(b) NNTSP matrix ordering
Figure 13: Examples of matrix reordering on graph presentation. (a) Using HDE algorithm. (b) Using NNTSP reordering. From [EDG*08], c) 2008 IEEE.

- Multiple synchronized views: These techniques link the matrix and node-link representation [HF06]. Both views show the same data and are synchronized during exploration. Thereby, the user can concentrate on whatever view is more suitable for the current task.

- Matrix with link overlay: The Matlink [HF07] approach enhances matrix visualization with links at the border of the matrix (connecting the nodes). Using link highlighting, the paths can be easily spotted in the Matlink view and at the same time, the advantages of the matrix representation are retained.

- Partial matrix and node-link representation: There are two main approaches. First, Nodetrix [HFM07] combines both representations in one view, where nodelink diagrams display the overall graph structure of the network, and adjacency matrices show communities. The work also discusses three ways of link display for this setting: aggregated links, underlying links, and underlying links with full size (Figure 15). These forms can be also used for attributed links. Secondly, layered graphs (directed acyclic graphs) can be represented by so-called 'quilts'. They arrange nodes in a matrix-like form and connect them with orthogonal edges. In this way, a clear view of the graph is created [WBS*08, BDF*10].

\subsubsection{Compound graphs}

Literature on visualization of graphs with hierarchic structure is relatively rare. We identify three main approaches.

Node-link graph visualization techniques: These use node-link diagrams for the lowest hierarchy level and then use 'bubbles' (enclosures) for various hierarchy levels. Examples include TugGraph [AMA09] and GrouseFlocks [AMA08]. The advantage of this method is its intuitiveness. However, for large graphs with many links, this view gets easily over-

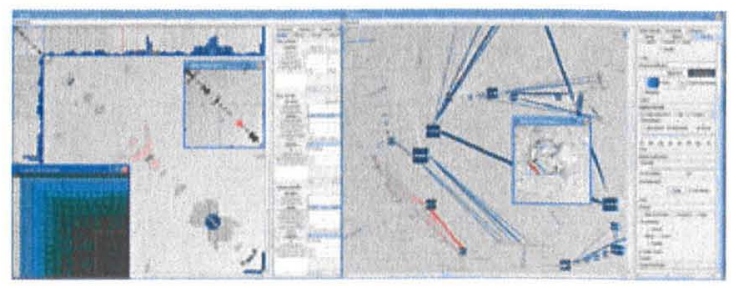

(a) Multiple linked views showing the same data using different representations

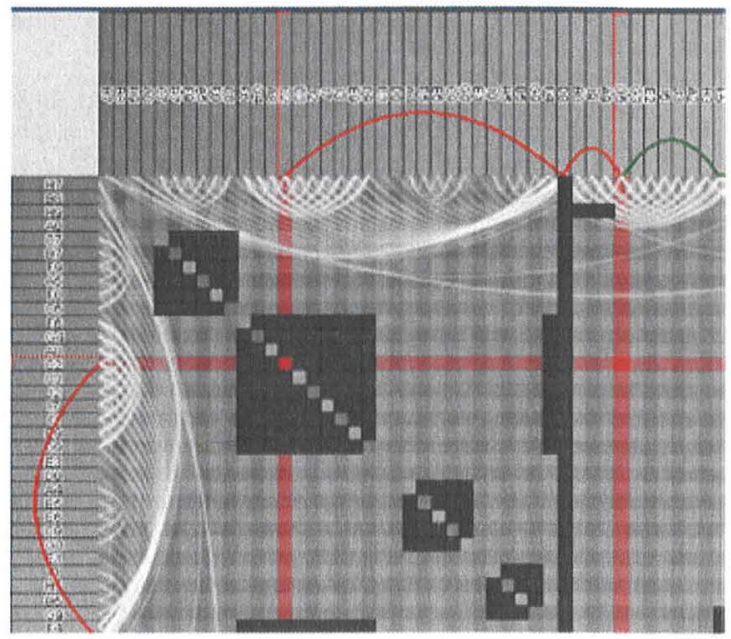

(b) Links connected to the matrix view highlighting paths between nodes

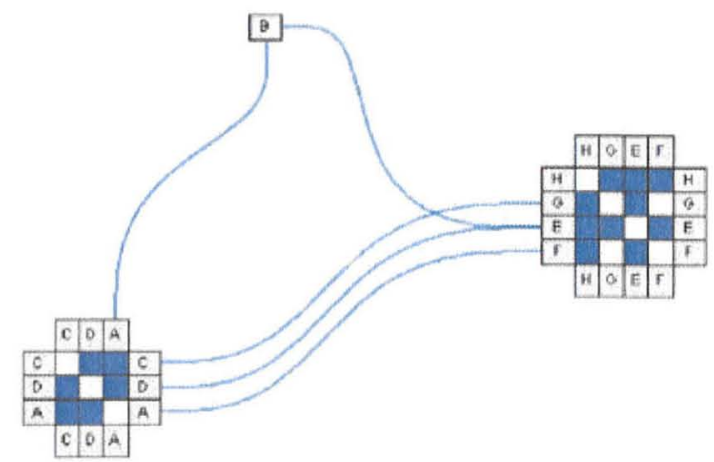

(c) Node-link and matrix combined showing dense areas as matrices (avoiding edge crossings)

Figure 14: Examples of combined matrix and node link graph visualization techniques. (a) Multiple linked views [HF06]. (c) 2006 IEEE. (b) Links connected to the matrix view [HF07]. c) 2007 Springer-Verlag Berlin Heidelberg. (c) Node-link and matrix combined - part and part [HFM07]. c 2007 IEEE.

crowded (Figure 16a). The edge over-plotting problem can be partially solved by edge bundling [Hol06] (Figure 12). Alternatively, only links between merged nodes can be drawn (Figure 16c). 


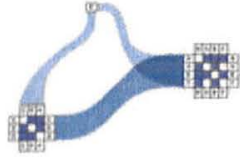

(a) Aggregated links

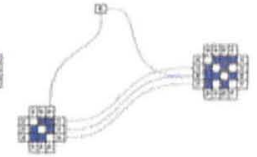

(b) Underlying links

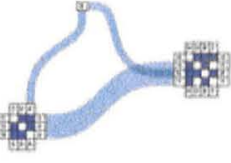

(c) Underlying links with full size
Figure 15: Three ways of link visualization in a combined node-link and matrix data representation using the NodeTrix approach [HFM07]. (c) 2007 IEEE.
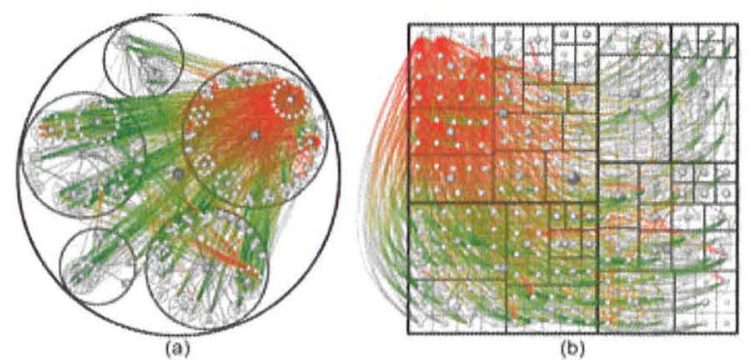

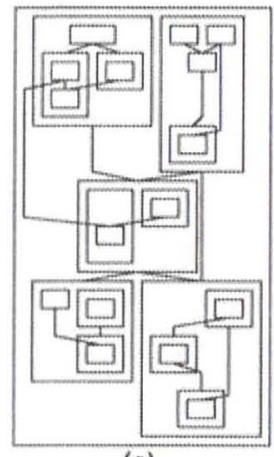

(c)

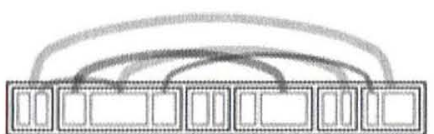

(d)

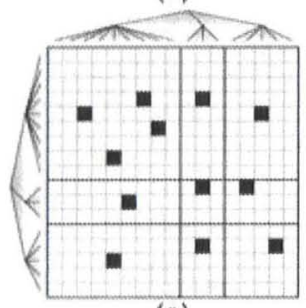

(e)
Figure 16: Visualization techniques for compound graphs [Hol06], c 2006 IEEE. (a) Node-link visualization with grouped nodes in 'bubbles'. (b) Links overlaying a treemap visualization. (c) Compound drawing using enclosures and links between merged nodes. (d) ArcTrees - links overlaying a ID treemap [BDJ05]. (e) A matrix view for showing relations between entities linked with tree view of the nodes as in MatLink approach [HFO7].

Treemap-based: A treemap visualization of the node hierarchy uses overlaid links between nodes [FWD*03] (Figure 16b). This approach may suffer from strong overplotting in case of many links between nodes of the hierarchy. Therefore, edge bundling is advised to improve the readability of the display [Hol06] (Figure 12). Similarly, also 1D treemaps with links between nodes, so called ArcTrees [BDJ05] can be employed (Figure 16d), but these do not scale well for large hierarchies.
Matrix view with links: These visualizations combine the generic node relationship visualization with a tree-based visualization of the hierarchic node relationships. This is an analogy to MatLink [HF07]. This view is very clear, however, it may be difficult to understand the compound relationships between nodes (Figure 16e).

\subsection{Visual representation of dynamic graphs}

In this section, we discuss two categories of visual display of the time changes on graph elements: using animation and using static displays. Animated displays usually employ or enhance static visualization techniques such as presented in Section 3.1. Animation is a natural way of conveying the change of the data over time. However, its effectiveness is limited by human perception capabilities. Usually, users are only able to recognize and remember larger changes in the data. Therefore, highlighting of graph changes is used. It allows for more effective spotting of differences between two successive time points [APP10]. The static view is preferred for more detailed analysis of data changes. Static views that also incorporate the time-dimension of the data are more complex. In the following, we categorize the visualization techniques according to the type of data changes captured into those that affect only data attributes, and those that affect also data relationships. Please note that visual analysis of changes in dynamic graphs is related to comparing graphs. Graph comparison is discussed in Section 5.2.

\subsubsection{Trees}

For the visualization of dynamic trees with only data attribute changes, either treemaps with time series in the leaf nodes [DHKS05, SKM06] or the so-called Timeline Trees [BBD08] can be used (Figure 17a and b). Timeline trees show the hierarchy on one side and the time sequences on the other side of the view. The treemap representation directly shows the hierarchic structure and time-variation in one combined view. This allows for an easy comparison of the time-developments across the hierarchy. However, the comparison is affected by different node sizes and difficult for small nodes. Therefore, a specific treemap layout preserving the aspect ratio has been developed [DHKS05, SKM06]. Timeline Trees assign the same space to all nodes. The vertical positioning of time lines allows for very good comparison of the values at the same time points. The separation of the time dimension from the hierarchic structure, however, complicates the comparison of tree branches.

For visualization of dynamic data with structural changes, animated views are used. Card et al. [CSP*06] have used and extension of DOI Trees [CNO2], [HCO4] to visualize the changes of an administration over time; a time-slider is used to control the visualized time-span. Animated graphs (Section 6.1) can be employed in general. In particular, the 


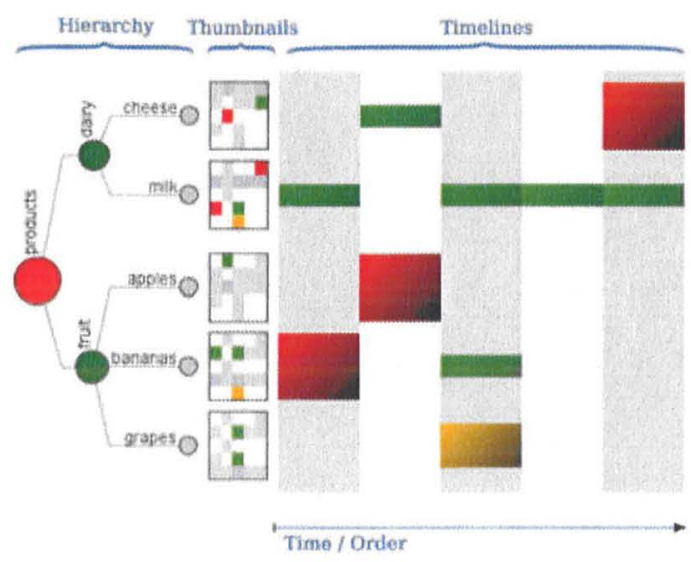

(a) Time line tree

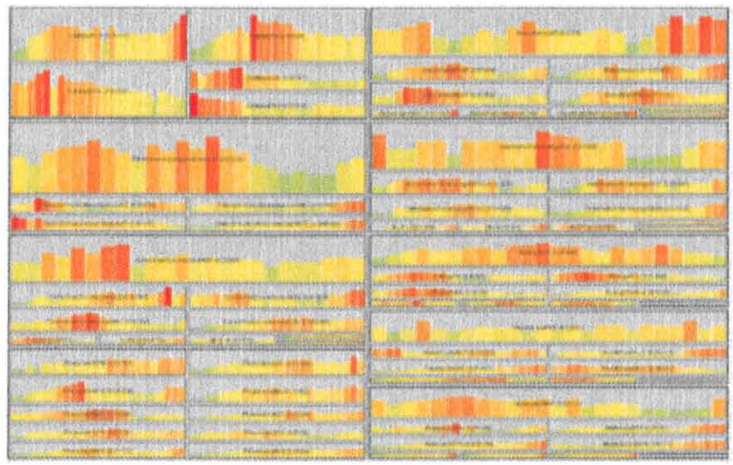

(b) Treemap with time series

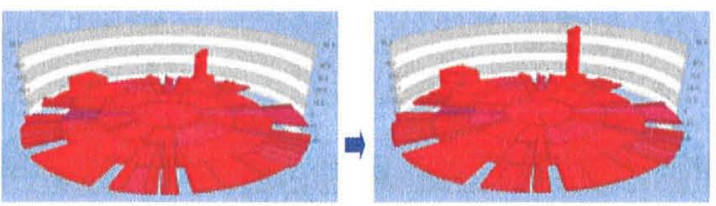

(c) Animated circular icicle plot

Figure 17: Visualization of time-dependent trees. (a) Time line tree [BBD08], c 2008 ACM. (b) Time series in the treemap nodes [DHKSO5], (c) 2005 IEEE. (c) Animated hierarchic circlular plots [TS08], c 2008 IEEE.

layouts based on the Sugiyama approach [GBPD04] are suitable. Alternatively, animated treemaps [GF01, TS07] or icicle/circular plots [TS08] can be used (Figure 17c). When choosing the graph layout, the layout stability needs to be taken into consideration. For example in the treemap representations, the spiral layout [TS07] achieves a high continuity with high stability of the layout. Strip and pivot-by-middle layouts have also been shown to have higher layout suitability [BSW02]. All these layouts are preferable in spite of their higher aspect ratios in comparison to the squarified treemap. Furthermore, dynamic Voronoi treemaps [SFL10] offer both good aspect ratios and stable layouts for displaying dynamic data. Alternatively, Tu and Shen [TS07] propose also static comparison of two time points in a treemap visualization (called contrast treemap).

\subsubsection{Directed and undirected graphs}

For attribute changes only, techniques for visualization of static graphs can be combined with visualizations of individual time-dependent data items (e.g. colour charts [SLN05]) are used (Figure 18a). The advantage of this approach is the large number of the available graph layouts.

In case of structural changes, time-dependent graph layouts (animated graphs) need to be employed [CBTT95, Nor96, DGK01, EHK*03, KG06]. In animated graph visualization (in analogy to animated tree visualization), a stable graph layout, which changes minimally, is of essence. A stable graph layout preserves the mental map of the user. It enables the user to follow changes on the screen [ELMS91, DGK01] and thereby it facilitates the analysis of graph changes. In laying out dynamic graphs, there is a large

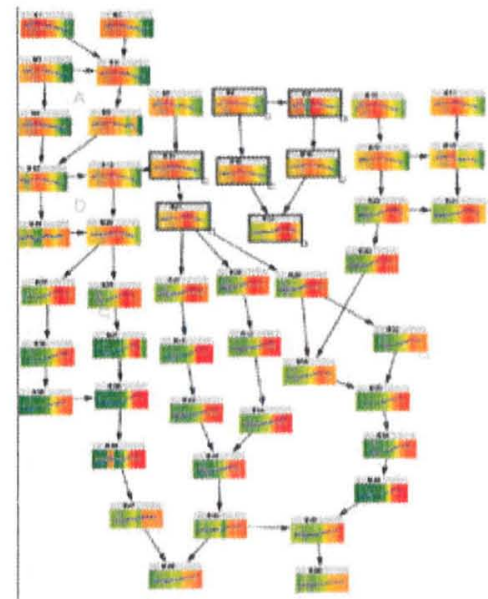

(a) Node-link diagram with time series in nodes

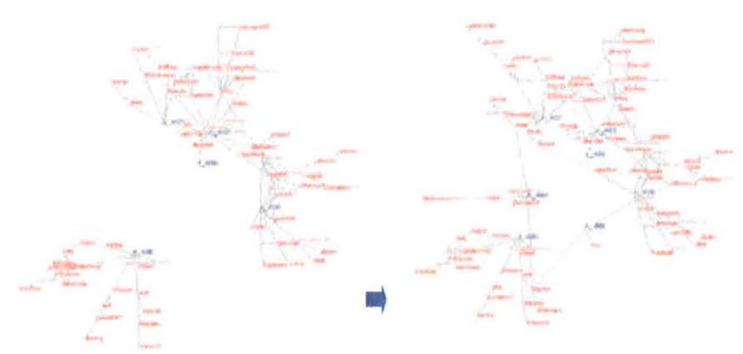

(b) Animated node-link diagram

Figure 18: Visualization of time dependent graphs. (a) Time series in nodes [SLNO5], c 2005 IEEE. (b) Animated graphs [FT08], c 2008 IEEE. 
difference between strategies for drawing graphs with known histories and those that need to be adjusted in real-time depending on new data streams. A paper of Frishman and Tal [FT08] addresses this particular issue by proposing an online algorithm for dynamic layout implemented on the GPU, thereby accelerating the layout computation (Figure 18b).

Instead of animation, Brandes and Corman [BC03] use the third dimension to show the evolution on time. GraphDice [BCD*10] uses interaction to switch between projections where time can be mapped to one dimension.

\subsubsection{Compound graphs}

There are only few techniques that visualize time-varying compound graphs. They employ either animation or static data representations.

Kumar et al. [KG06] present a specific layout for animation of a node-link diagram with transparent 'bubbles' for the hierarchic grouping of nodes (Figure 19a). Frishman and Tal [FT04] present a layout which focuses on maintaining the clustered structure during the animation. The groups of nodes are displayed using bounding boxes around the groups. Reitz et al. [RPD09] use dynamic graph layouts for showing areas of interest in dynamic compound graphs.

A static approach to visualization of dynamic compound digraphs using TimeArcTrees was presented by Greilich $\mathrm{et} \mathrm{al}$. [GBD09] (Figure 19b). They show a sequence of node-link diagrams with horizontal node alignment in a single view, thereby supporting their direct comparison. TimeRadarTrees [BD08] use radial tree layouts for the hierarchy and a sequence of circle segments for representation of the temporal change of the structure (edges) of the Digraph (Figure 19c). This view easily gets complex for larger graphs.

\section{User Interaction in Graph Visualization}

Interaction helps users solving tasks connected to exploration of graphs. These tasks can be of different nature such as topology-based or attribute-based [LPS*06]. Topologybased tasks include finding adjacent nodes, or determining connections between nodes. Attribute-based tasks include, for example searching for nodes with specific values, and finding edges of certain types. For each task, one or more interaction techniques can be employed. Standard interaction techniques such as zooming, panning or brushing and linking [CMS99, War00] are commonly used in graph visualization. In addition, specialized techniques have been developed for interactive visual graph navigation and exploration.

Interaction and exploration are deeply inter-related. Some graph analysis systems such as Pajek [dMB05] claim to support exploratory graph analysis by chaining complex operations on graphs without showing the intermediary results. However, Ahlberg et al. describe interactions and more

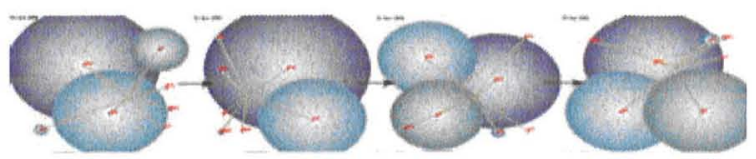

(a) Animated compound graphs

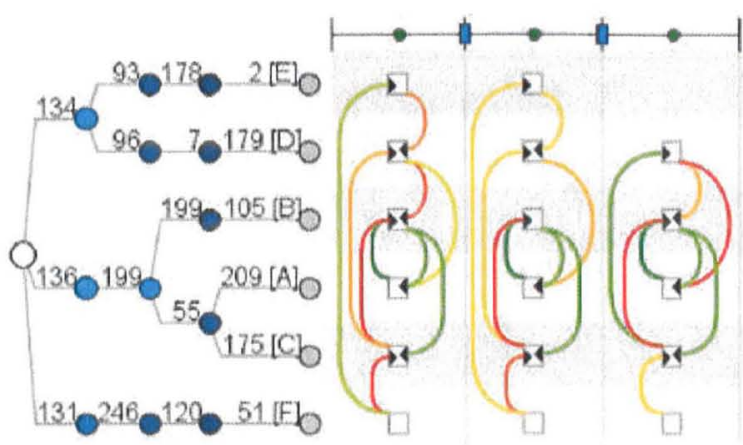

(b) TimeArcTrees

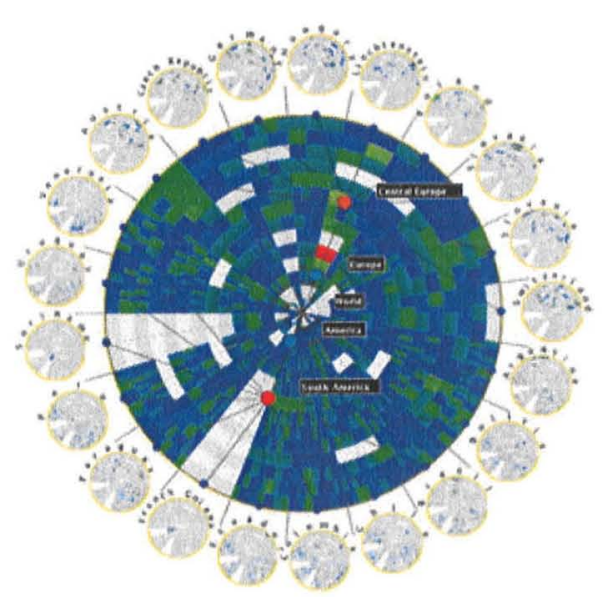

(c) TimeRadarTrees

Figure 19: Visualization of time varying compound graphs. (a) Animated graphs by Kumar et al. [KG06], c 2006 IEEE. (b) TimeArcTrees [GBD09], c 2009 held by the authors. (c) TimeRadarTrees [BD08], c) 2008 held by the authors.

specifically dynamic queries [AWS92] as required to truly achieve exploration. The main reason is cognitive: exploring requires several hypothesis to be maintained in short-term memory which is very limited in capacity. Planning complex operations without feedback or using a textual syntax consumes all the short-term memory and exploration becomes impossible from short-term memory alone. Therefore, providing interactions with immediate feedback for the most frequent operations supports exploration. Other less frequent operation could still be done using more complex mechanisms, as explained in the next section on graph analysis. 
The categorization of interaction techniques can be based on various criteria such as task, user intention [YKSJ07] or user action [EF09]. These criteria are interrelated. For example, one task may include performing several actions, or one task may correspond to several user intentions. Moreover, one user intention can be achieved by several user actions or, vice versa, an action can suit several intentions.

We categorize interaction techniques according to stages in the Information Visualization reference model of Card et al. [CR98], [CMS99] and user actions. The reference model has three stages: data, visual form (a.k.a visual abstraction) and view. The classification criterion is whether the user action affects the data (the selection of the displayed data or the data values), the visual display of the data (visual parameters or visual representation), or the view. Data, visualization and view manipulation can be used for interactive data exploration and navigation. This categorization follows the idea of Elmqvist and Fekete [EF09] and Bertini and Lalanne [BL09]. Please note that these three types of interaction are sometimes closely connected. For example, data manipulation may automatically lead to changes of visual parameters (e.g. data filtering can influence the graph layout, or zooming can be combined with data filtering forming a type of semantic zooming).

\subsection{View interaction}

\subsubsection{Panning and zooming}

Panning and zooming allow to navigate in any direction and change the zoom-level in the view. For node-link diagrams, a specific type of panning (guided panning) has been proposed. It allows to navigate along edges of a selected node and thereby to explore the structure of the graph. It can be combined with automatic zooming on the edge and distortion of end-node position closer to the currently selected node $\left[\mathrm{MCH}^{*} 09\right]$.

\subsubsection{Magic lenses}

Owing to the limited display space, showing the whole data set may lead to strong over-plotting or very small (up to, unreadable) data items. Magic Lenses [BSP*93], including distortion techniques, change the representation or allocate more space to items in focused areas and thereby, improve the readability of the data of interest. They are used both for node-link and space filling graph visualization techniques. The changes can concentrate either on one area or on multiple areas of the screen. For geometric changes, the technique is called fisheye views. Interactive selection of the focus area helps to explore different parts of the data in more detail.

- Single focus: Graphical fisheye views were introduced in [SB92]. So-called edge lenses resolve strong overlaps of

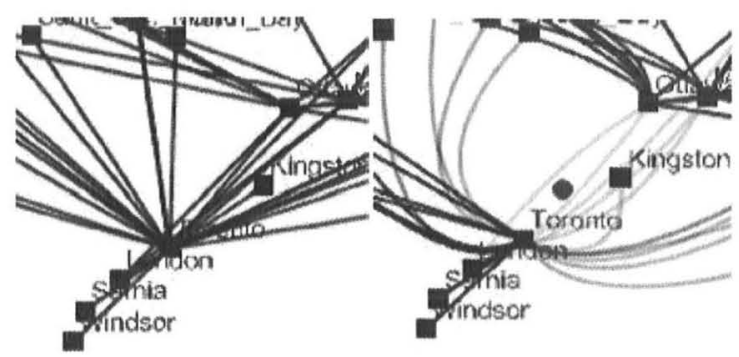

(a) Original view

(b) Edge lens

Figure 20: Example of edge lens interaction. (a) Original view without lens. (b) Using edge lens From [WCG03], c 2003 IEEE.

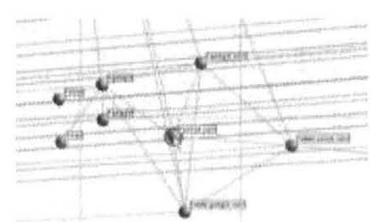

(a) Original view

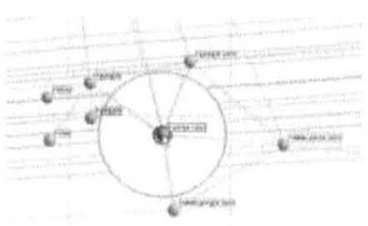

(b) Local edge lens

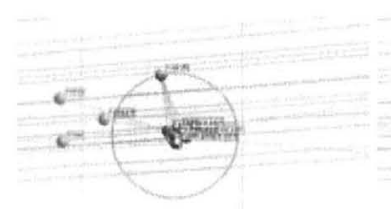

(c) Bring neighbors lens

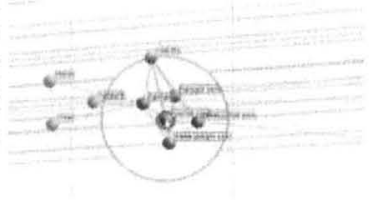

(d) Composite lens
Figure 21: Examples of different types of edge lens interactions. (a) Original view without lens. (b) Using local edge lens. (c) Using bring neighbors lens. (d) Using composite lens which combines (b), (c) and Fisheye lens. From [TAvHSO6], (c) 2006 IEEE.

edges in the view. They displace the edges to a larger area [WCG03] (Figure 20). This approach is especially useful for geographic-based graphs, where node repositioning is not desired and therefore, cannot help to solve edge overlap. Another approach uses filtering of interesting edges in a specified area, or moving neighbour nodes closer to a selected node relying on the graph structure [ $\left.\mathrm{MCH}^{*} 09\right]$. This type of node position change can be combined with geometric view distortion [TAvHSO6] (Figure 21). In node-link visualization of hierarchies, a degree-of-interest function can be used for allocating more area to more interesting parts of the tree, for example in DOITrees [CNO2, HC04].

None-geometric magic lenses include Excentric Labels and Colour Lenses. Excentric Labels [FP99, BRL09] show labels or other statistics for items contained in dense focus regions (nodes or matrix cells). The information 


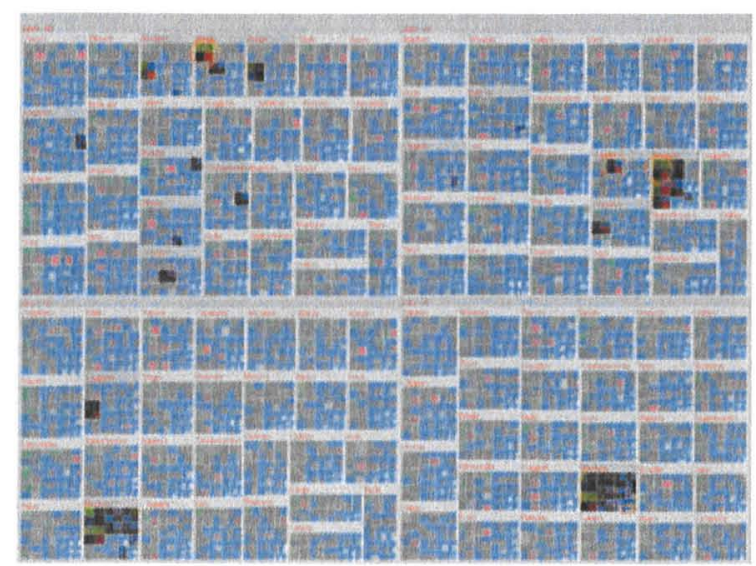

(a) Original view

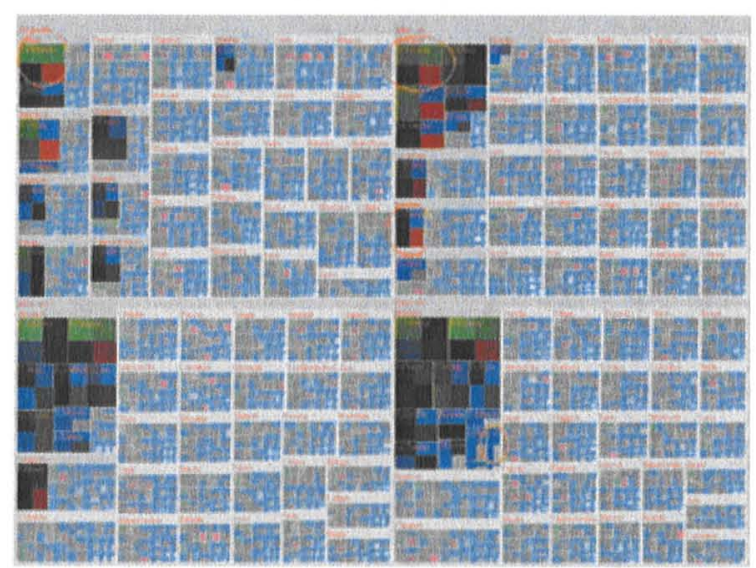

(b) Balloon focus

Figure 22: Multiple foci in a treemap. (a) Original view. (b) Using balloon focus. From [TS08], c 2008 IEEE.

is displayed outside the focus region with connectors linking the nodes/cells to their related label. Colour lenses [EDF10] dynamically adapt the colour range of items inside the focus region to better use the screen colour range when mapping values with a very large dynamic to the colour of nodes or matrix cells.

- Multiple foci: Multiple foci distort several view areas at the same time. It is useful for comparing various parts of the display or focusing on several items that are spread across the view. In node-link diagrams either magnification of the areas of interest [SZG*96, TS99] or space folding (shrinking of area out of focus) can be used [MGT*03, ERHF09] (Figure 28, bottom right). For treemaps, the so-called balloon focus can be used for enlarging multiple items in a treemap [TS08]. This approach keeps the form of other areas keeping relative position of items unchanged (Figure 22).

\subsection{Visual abstraction interaction}

In these approaches, the change of the visual presentation of the data concerns adjusting the type of visual presentation and its parameters.

Most of the graph visualization systems provide standard dialog boxes and widgets to change the visual abstraction parameters, including the layout technique and its various parameters. Currently, very few systems allow the interactive manipulation of layout parameters, except using indirect manipulation such as sliders, list boxes, radio buttons and check boxes. Rich visualization systems provide a large number of these indirect manipulation widgets which use an important amount of the screen real-estate and force users to search for the right widget by reading their labels and trying to make sense of them, which can be quite long and tedious. This is why several research work is devoted to providing more direct mechanism to change the parameters.

\subsubsection{Changes of visual parameters}

These techniques affect the parameters of the visual presentation. They include highlighting of items and other techniques.

Highlighting: The emphasis of interesting items is a standard interaction technique. Recently, new techniques for highlighting a node and its neighbourhood using hotbox and lasso selections were presented in [MJ09].

Brushing and linking: Multiple coordinated views are used to show the data from different perspectives. In these views, changes in one visualization (e.g. highlighting) are automatically transferred to the other views. For example, a matrix view coupled to a hierarchical view of the data can be used to reveal important information in the data [AvH04].

Semantic zooming: Semantic zooming combines zooming with an increasing level of detail. In particular, graph aggregation can be used for gaining a coarser view on a large graph. The semantic zooming increases the level of detail by drilling down to lower levels of aggregation of the original data [EDG*08, AvH04].

\subsubsection{Changes of visual scheme}

Changes of the visual scheme cover changing of the type of data visualization either by changing the layout or by changing the visual mapping.

Layout change: In node-link diagrams, layout change (adjustment) affects the positions of the data items on the screen (Section 3). It can be performed by changing of the layout type with automatic recalculation of the new layout, by manual movement of nodes or by adjusting the layout parameters including automatic readjustment of the layout. 


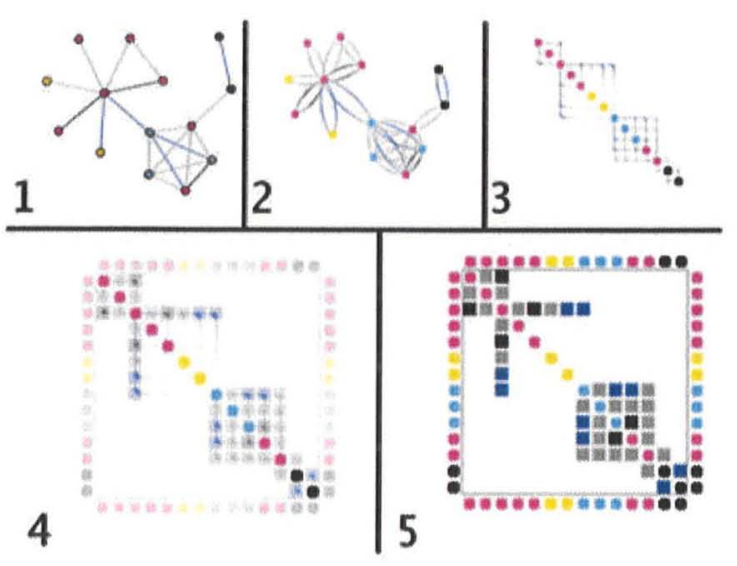

Figure 23: Transformation of visual representation of a graph from node-link to matrix view. The picture shows five stages of this process [HFM07], c 2007 IEEE.

When concentrating on user-defined changes to graph layouts, an approach to easy selection and layout change of nodes and subgraphs was presented in [MJ09]. Furthermore, interactive adjustment of the layout constraints was presented in [DMW09]. For matrix visualizations, userdriven reordering of matrix representation was described in [HF06].

Change of visual representation: The change of the type of data presentation, for example from a matrix to a node-link diagram was presented in [ZMC05, HFM07]. This change can affect the whole data view [HFM07] (Figure 23) or only a part of it [ZMC05, HFM07]. By changing the visual representation, new insights into the data can be reached. To be able to follow the changes, smooth animations across transitions should be used.

\subsection{Data interaction}

Data-level interaction affects the selection of the data to be displayed, or may change the data values and structure.

Some operations can be done interactively but general graph analysis system provide more sophisticated mechanisms including scripting languages or powerful macro facilities to perform more complex operations.

\subsubsection{Data filtering}

These interaction techniques influence which parts of the data set are displayed. The data filtering may follow three paths.

A top down approach: This approach starts from the whole graph and then constrains the part of the data set to be visualized by filtering according to criteria or by manual data selection. The disadvantage of this approach is the need to show the whole graph at the beginning, which may require higher computational time for the layout and may lead to occlusions owing to the limited screen size. The advantage is gaining an overview of the graph structure first and then concentrating on interesting parts.

A bottom up approach: This approach starts from one selected node [Fur86, AF07, vHP09] and successively shows more nodes/connections on demand. There are two main methods of choosing the additional nodes/edges to be displayed: based on graph structure or based on a degree-ofinterest function. The advantage of this approach is that only the most interesting part of the data set is visualized, however it is difficult to determine the starting point for the exploration and to define the degree-of-interest function. Therefore, we consider these methods in more detail.

- Navigation based on graph structure: These techniques reveal/hide that part of the graph that is determined by the connections between nodes. In graphs, neighbourhood traversal shows neighbour nodes of a focus node up to a certain level [HB05]. For hierarchies, several traversal methods for have been described in [EF09]. The hierarchy traversal methods include: (1) above traversal, where nodes up to a certain level are shown; (2) below traversal, where nodes starting from a selected level are displayed; (3) level traversal, where nodes at a certain level are displayed; (4) range traversal, where nodes in a range of levels are shown and (5) unbalanced traversal, where certain branches of a tree are visible (Figure 24).

- Navigation based on a degree of interest function: These methods start from a selected node, and next the edges and nodes of highest interest are shown [Fur86, vHP09]. For the determination of the interesting nodes, a specific degree of interest (DOI) function is used. Depending on the specification of the DOI function, various graph exploration paths can be followed. These DOI functions were used for building specific views on trees (DOITrees,SpaceTree) [CN02, HC04, PGB02]. In the work of Furnas [Fur86], the DOI of a node depends on the distance to the node in focus and the a priori interest in this node (e.g. according to node importance in the network, or node properties). Van Ham and Perer [vHP09] extended this function with user interest (UI), which reflects the current specific exploratory focus of the user.

A middle-out approach: This method combines both bottom-up and top-down approaches. It starts with a coarsened graph (middle) and then interactively either reduces or increases the graph coarsening level by hiding visible nodes or showing additional nodes [WMC*09]. For determining 


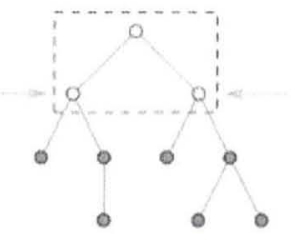

(a)

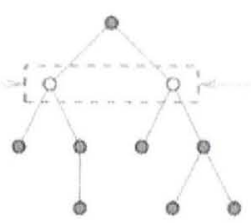

(c)

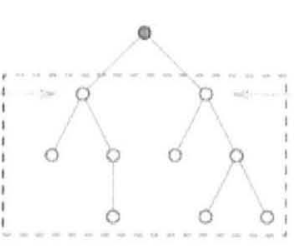

(b)

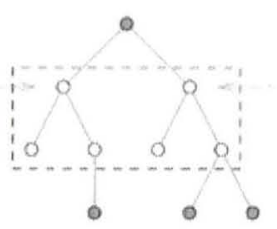

(d)

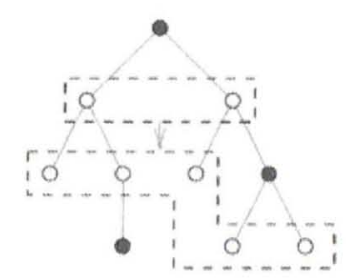

(e)

Figure 24: Hierarchy traversal strategies [EF09], (c) 2009 IEEE. (a) Above traversal, (b) below traversal, (c) level traversal, (d) range traversal and (e) unbalanced traversal.

the middle coarsening level and the next interactive steps, graph algorithms are used (Section 5).

\subsubsection{Changes of data values}

In these approaches, the change of the displayed data set result from direct data value manipulation. Specifically, the user can change the data values on one level or create/change graph aggregations.

Graph editing: The user can interactively delete or add nodes or edges directly in the visual interface. These graph editing actions trigger adjustment of the layout, while still maintaining the layout style and, where reasonable, the current layout topology. Graph editing affects the structural properties of the graph. In particular, the changes can affect specific types of subgraphs (so-called motifs). Automatic identification and highlighting of such structural changes was presented in [vLGRS09].

Interactive graph aggregation: For simplification of graphs, graph aggregation is often used. The graph aggregation can be pre-defined, or determined interactively by the user [HF06, AMA08, AMA09]. For example, GrouseFlocks

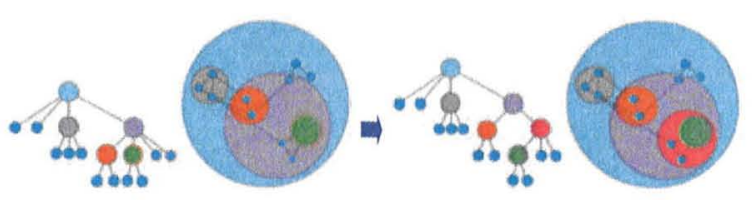

(a) Creating new aggregation node

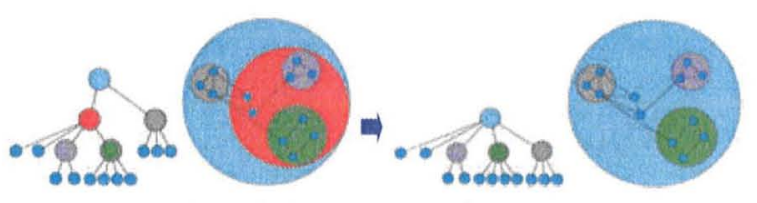

(b) Deleting an aggregation node

Figure 25: Interactive editing of a graph hierarchy. (a) Creating a new aggregation node by merging of nodes. (b) Deleting an aggregation node, thereby revealing the underlying merged nodes. From [AMA08], c 2008 IEEE.

[AMA08] allows the user to add and remove aggregated nodes on demand (Figure 25). This allows for variable views on the graph and its structure.

\section{Graph Analysis}

Algorithmic graph analysis is beneficial during all stages of the visual graph analysis process. Relevant techniques allow, for example to reduce a large graph to a smaller graph prior to visualization, to search for specific graph structures of interest or to find similarities and dissimilarities for generating comparative graph views. In this section, we describe a number of graph analytical approaches.

\subsection{Analysis of graph structure}

In most user tasks, the analysis of the relationships between entities in the graph and the assessment of the global graph structure plays the key role. These tasks may be effectively supported by a combination of algorithmic graph analysis and interactive visualization. The algorithmic methods allow, for example to calculate node/edge properties, identify clusters in the graphs, etc., the results of which are visualized interactively. In the following, we summarize the methods according to user tasks starting from more simple to more complex tasks.

\subsubsection{Identification of important nodes}

In networks, some nodes play a specific role owing to their position within the network. For example, so-called hubs and authorities can be identified and visualized in the network, enabling faster analysis of the graph [OPPROG09]. The importance of nodes and edges is measured by derived quantities (i.e. network metrics) such as centrality-based measures 


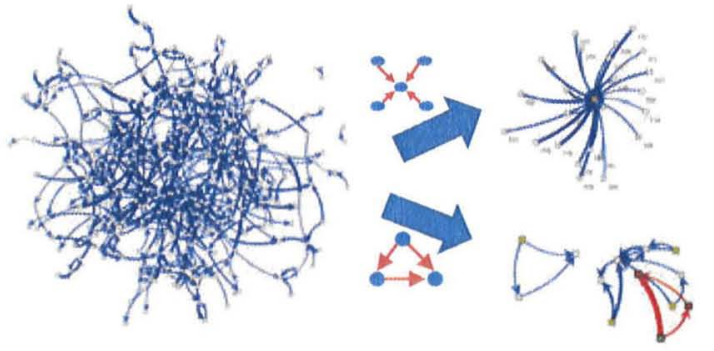

Figure 26: Interactive graph motif search and visualization. From [vLGRS09], c 2009 held by the authors.

[Fre79] and ranking-measures [WS03]. Network metrics can help the analysts to explore networks. Color coding of nodes or edges by metric values, or displaying metrics and networks in multiple linked views (as lists, scatterplots or parallel coordinates) are used in this respect. They offer the possibility to interactively chose the metrics of interest and to filter/highlighting nodes according to these metrics [CJM04, PS06, BCD*10, VMCJ10].

\subsubsection{Analysis of connections between two nodes}

Besides focusing on single nodes, relations between two nodes can be analysed, typically by calculation and highlighting of shortest paths between the entities. Usually, such analysis is combined with interactive selection of two entities of interest [HB05, HF07, TK08, GBD09] (Figure 14b).

\subsubsection{Analysis of graph substructures}

In many applications, specific types of substructures (i.e. motifs) play an important role. For example, in social networks, cliques identify highly connected communities, or feed-forward motifs (substructures in form of a triangle where directed edges exist from nodes $\mathrm{A}-\mathrm{B}, \mathrm{A}-\mathrm{C}$ and $\mathrm{B}-\mathrm{C})$ in biologic networks indicate the functional properties of the network [Sch08]. To support the substructure analysis, these motifs can be calculated and visualized in the network [MMO05, SS05, KSS06, vLGRS09, MJW*09] (Figure 26). The type of structure can be interactively chosen by the user in order to support various analytical tasks.

\subsubsection{Analysis of graph structure on several aggregation levels}

User-defined or data-driven graph aggregation can reveal relationships between groups of entities in a graph. The grouping may be based on categoric node attributes [Wat06], or on a pre-defined node hierarchy [AMA09]. It can also be user-specified [AMA08], on clustering results based on node properties [PS06], or depend on structural properties of the graph [vLGRS09] (Figures 5 and 25).

\subsubsection{Identification of the impact of graph changes on the structural properties}

In time-dependent graphs, the role of the nodes can change over time, therefore analysis and visualization of topologic properties (e.g. betweenness centrality) of selected nodes has been proposed [PD08, PRB08]. In addition, when analysing user-defined changes (in what-if-scenarios) the impact of node or edge deletion/addition on local substructure can be analysed and highlighted [vLGRS09].

\subsection{Graph comparison}

One specifically important analytical task is the examination of the similarities and differences between multiple graphs, especially focusing on structural aspects. Usually, structural differences are in the focus. Such difference may be identified by the identical node labels in both graphs, or by graph matching algorithms. After the matching, visualization is employed to explore the differences [AWW09]. There are various types of analysis which we describe next.

\subsubsection{One-to-one node comparison of two graphs}

Probably the most common task in graph comparison is the matching of individual nodes from one graph to individual nodes of the second graph. The VisLink visualization approach [CC07] was developed to support this task. It shows both graphs on separate planes in $3 \mathrm{D}$, and draws matching links between corresponding nodes (Figure 27a). For comparison of hierarchies, a similar approach, based on drawing the two hierarchies in opposite parts of the display and linking of their leaf nodes was proposed in [HvW08] (Figure 27b). In both cases, the visibility of matching links can be increased by edge bundling.

\subsubsection{One-to-many nodes comparison of two graphs}

One-to-many nodes comparison concerns correspondence of one node in one graph to many nodes in another graph. Di Giacomo et al. [GDLP09] developed a system that visualizes these one-to-many connections with low overlapping of links (Figure 27c).

\subsubsection{Structural differences between two graphs}

When analysing structural differences between two graphs, analysts are often interested in identifying which links or parts of the graphs correspond to or differ from the other one. For the analysis of trees, the TreeJuxtaposer system 


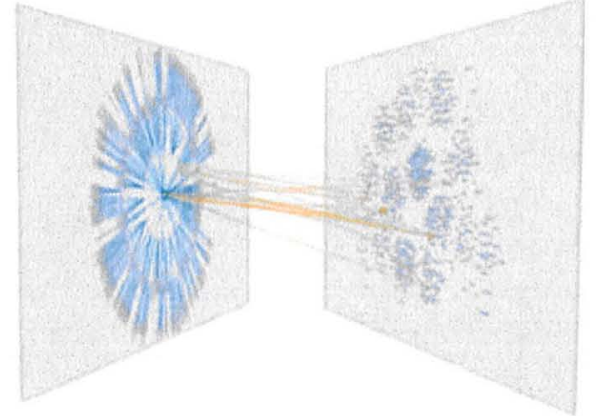

(a) One-to-one graph matching

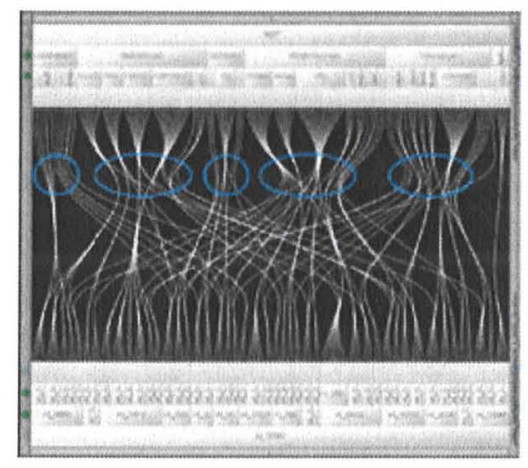

(b) One-to-one hierarchy matching

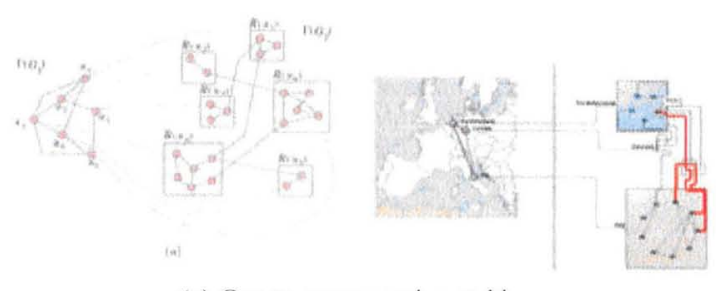

(c) One-to-many graph matching

Figure 27: Visualization of graph comparison. (a) One-toone graph matching [CCO7], (c) 2007 IEEE. (b) One-to-one hierarchy matching [HvW08], c 2009 held by the authors. (c) One-to-many graph matching [GDLP09], c 2009 SpringerVerlag Berlin Heidelberg.

supports to analyse and highlight structural differences between two trees [MGT*03] (Figure 28). For general graphs, Fung et al. [FHK*09] use both multilevel graph views following the VisLink approach [CC07], and overlapping of two networks with highlighting of common structural parts (Figure 29a). Archambault [Arc09] uses graph aggregation and graph filtering to reveal structural differences between two graphs (Figure 29b).

\subsubsection{Structural similarity among multiple graphs}

Structural comparison of multiple graphs is often based on their description by several graph properties such as graph

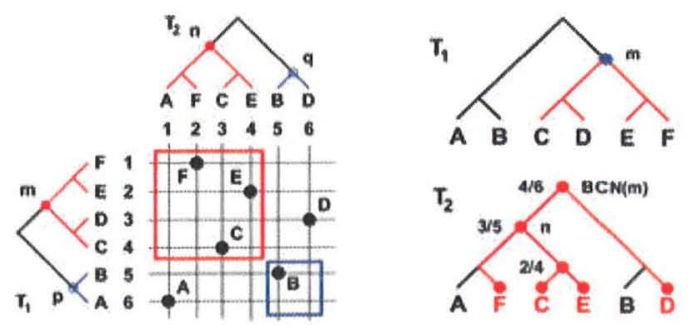

(a)

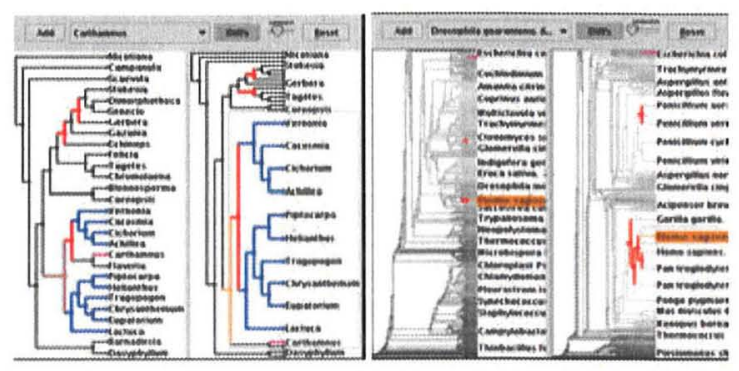

(b)

Figure 28: Tree comparison. (a) Schema of the tree comparison. (b) Example of tree comparison using highlighting of tree differences. The left view shows the traditional view, the right view is distorted to emphasize important parts of the tree [MGT*03], c 2003 ACM.

size, density, connectedness, etc. (see also Section 2.1). These properties can be used for exploration of large sets of graphs [FPSG10], or for the determination of structural similarity between graphs. Graph similarity may serve as an input for clustering of graphs (grouping similar graphs). Clustering helps gaining overview of types of graphs in large graph databases. Interactive combination of graph clustering and visualization of clustering results has been proposed in [vLGS09] (Figure 30).

\section{Concluding Remarks and Future Challenges}

Research on visual graph analysis deals with the interrelated issues of graph drawing, graph presentation, human-computer interaction and analytics. This state-of-theart report represents an encompassing overview and systematization of recent developments in this field. Many advances have been made on individual parts of visual graph analysis. On the other hand, the surveyed literature discusses many important open challenges, that researchers see in need of work. In the following, we summarize key research challenges. The discussion of the relevant topics is divided into three broad areas: graph visualization and interaction, visual analysis systems and conceptual issues. 


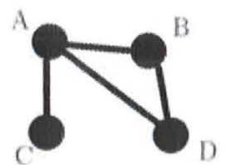

Graph $G_{1}$

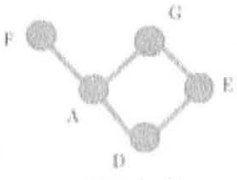

Graph $G_{2}$

(a)

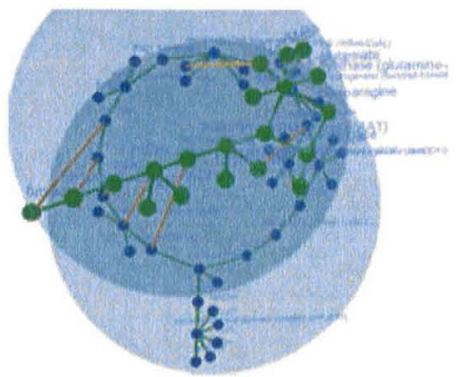

(b)

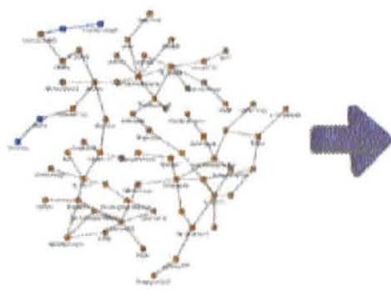

(c)

Figure 29: Visualization of structural differences between two graphs. (a) A schematic illustration of graph difference. (b) Visualization of graph differences using network overlapping [FHK*09], co 2009 IEEE. (c) Visualization of graph differences using difference hierarchies [Arc09], c 2009 held by the author.

\subsection{Graph visualization and interaction}

\subsubsection{Scalability issues in graph drawing}

There has been much interest in the development of faster layout algorithms that produce more readable layouts for large graphs, also using parallel computing, as provided, for example by current CPUs and GPUs. It is recognized that using a combination of automatic graph layout generation and user-oriented, interactive layout steering, better layouts can be obtained. As graphs get larger, graph filtering and aggregation have been the main means of graph simplification allowing to draw them. Alternatively, the limited screen space leading to strong over-plotting in large graph visualization can be avoided by drawing graphs on large screens, where specialized layouts can be applied [MGL06]. It can be foreseen that work on more sophisticated graph layouts revealing the main structures in the whole graphs, or parts
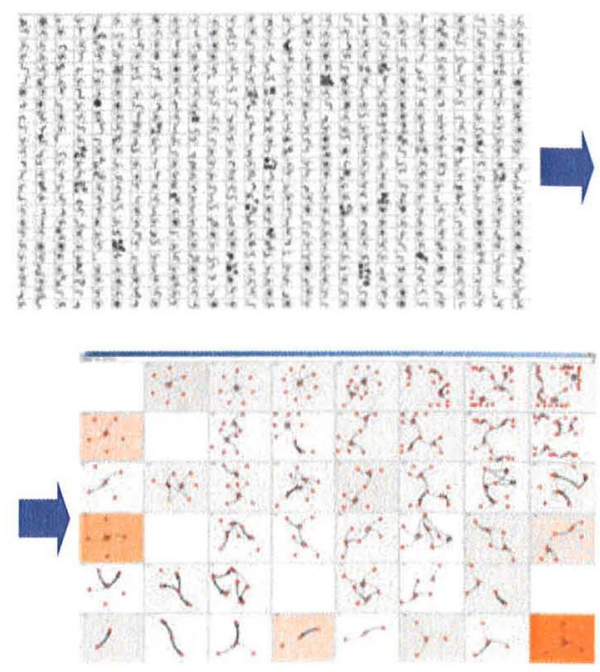

Figure 30: SOM-based graph clustering for analysis of types of graph data space and similarities between graphs [vLGSO9], c 2009 IEEE.

thereof, will continue. In particular, user involvement in the graph layout process involving analytical expertise of the user is a promising approach and may lead to easier interpretation of the drawings.

From an analytical perspective, also the understanding of the meaning of the nodes and edges, besides their global structure, is necessary. In particular, the readable/nonoverlapping drawing of nodes, edges and their labels is an important issue. When displaying graphs with labels, even smaller graphs can easily lead to overcrowded displays. This topic is gaining more interest in visual analytics research.

\subsubsection{Graph types in graph drawing}

In recent years, the variety of considered graph types has increased substantially. In particular, there has been a large amount of work on drawing dynamic and compound graphs. When drawing dynamic graphs, layout stability and on-line graph drawing are the main points of interest for the future research. In visual analysis, the understanding of the graph changes needs to be supported by stable layouts that preserve the mental map of the analyst thereby allowing them to follow changes on the screen [DGK01]. These layouts should be very stable for minor graph changes and, at the same time, be able to effectively show large graph changes. Although a non-trivial challenge, if successfully supported it may lead to easier spotting of structural changes in the graph and thereby, more efficient and effective analysis. On-line graph drawing, where the data stream is unpredictable, poses major challenges in this respect. 
Compound graphs as a combined graph type, including aggregated graphs, represent a complex data type. The main analytical problem there is the understanding of both types of connections in a graph, as well as the understanding of the graph structures on multiple abstraction levels. This is a very cumbersome task, which can be supported by graph visualization systems. However, the drawing of such complex graphs is still in its infancy.

In the future, also further graph types such as hypergraphs [KKS09], or graphs with overlapping sets of nodes [HD10] may become more prominent in visual graph analysis research.

\subsubsection{Graph uncertainty}

Graph visualization by now mainly deals with drawing graphs with given data, largely disregarding graph uncertainty. Visualization of uncertain data is a general challenge in visual analytics. As has been shown in [GS05], the degree of data certainty affects analytical decisions. Therefore, it is an important issue in visual graph analysis. In graph visualization, various types of uncertainty can be regarded. The uncertainty can relate to the graph structure (the existence of nodes and edges between them) and/or on graph attributes (edge and node attributes). For displaying node and edge attribute uncertainty, various methods from multivariate data visualization with uncertainty (see, e.g. overviews given in [PWL97, THM*05, GS06]) could be applied. However, their applicability to graph visualization needs to be studied. When dealing with structural uncertainty, there are few dedicated techniques. For example, CandidTree [LRCP07] uses transparency and colour for conveying uncertainty in merged graphs. Therefore, it is expected that more methods will be developed in the future to address graph uncertainty issues.

\subsubsection{Perception issues in graph visualization}

The understanding of graph structures in visualization strongly depends on human perception capabilities. Studies of human perception for graph drawing have recently focused on comparison of graph understanding using varying graph layouts. In graph design, studies on edge visualization have shown that the edge design has an influence on the graph reading. These various studies have given rise to new problems in graph visualization, which need to be studied in the future.

\subsubsection{Graph interaction techniques}

In graph exploration, recently new interaction techniques for various graph types have been developed. These techniques increasingly make use of the structural properties of the graph to interactively navigate in the graph (e.g. [TS08, vHP09,
TAS09]). This tendency supports the analytical purpose of graph visualization, as analysts can more easily examine the structural relationship between entities in the graph. In the future, this direction can be extended.

\subsection{Visual analysis systems}

\subsubsection{Visual analysis systems}

In line with Keim's visual analytics process [KAF*08], modern visual graph analysis systems should interactively integrate data pre-processing, interactive data visualization and building and visualizing of data models for gaining knowledge from the data. Many visual analysis techniques already include parts of this process. However, many of them rely on black box computations (e.g. automatic graph preprocessing, automatic calculation of graph similarities or cliques). To support the variable hypothesis-insight-driven analytical process, more user involvement in the process should be aimed at. The user should have full control of the type of the analysis and its parameters. As this process includes multiple loops, interactive feedback possibilities are necessary. Therefore, integrated visual analysis systems should include such features.

\subsubsection{Integration of various data types in visual analysis}

Graphs as data structures capturing relationships between entities are part of a larger set of data types examined in various applications. Usually, the analysis of graphs is undertaken in combination with analysis of related data sets, or other data sets are transformed into graphs for their analysis [CGK*07, BMGK08]. For analysis of the various data sets as a whole, the sole focus on visual graph analysis (in particular graph exploration) without taking other relevant data into account, is not suitable. In the future, larger integrated visual analytics systems combining research results from several areas are needed.

\subsubsection{Addressing new analytical tasks}

With the increasing data set sizes and their complexity, new analytical tasks arise. For example, one such task is the examination of the similarities and differences between graphs. This task builds on the examination of the structure of one graph as discussed above. Lately, several papers about visual graph comparison for both trees and general graphs have been published (Section 5). The comparison can concern only two graphs, trying to match nodes and edges between them. It can focus on finding similar graphs for one particular graph from a large set of graphs. It can concern gaining an overview of the types of structures in a large set of graphs. It can concentrate on analysing the similarities of whole graphs or on matching of parts of one graph to other graphs. Owing to its complexity, and the variety of the 
problems, it is foreseeable that the research in this area will need to continue.

\subsubsection{Collaborative visual graph analysis}

For solving complex analytical tasks concerning multiple large related data sets, a collaboration of several experts is necessary. Recently, the development of collaborative visual analysis systems has received attention $[\mathrm{BMZ} * 06, \mathrm{Kee} 06$, ITC08]. However, collaborative visual graph analysis is not represented prominently. Therefore, the study of collaborative systems including graph data sets would be of advantage. The specifics of graph exploration, in particular, need to be studied.

\subsubsection{Insight provenance for visual graph analysis}

In Visual Analytics applications, the analytical processes are often long-running and/or distributed. To support the reproducibility, reversibility and automation of these processes, user tracking of the graph interaction steps is necessary. As a basis for tracking, a taxonomy of graph interaction techniques is necessary. The theory of interaction is a general Visual Analytics challenge [TC06]. Although several interaction taxonomies also for insight provenance have been recently introduced [GZ08, HMSA08], their applicability and the need for their adaptation to graph analysis needs to be studied. In return, specific classifications of graph interaction techniques could be developed. In this report, we have aimed to classify them for gaining a concise overview of the current state of the research. This classification, however, may not be directly applicable to user tracking applications.

\subsubsection{Applications}

For analytical purposes, standard graph visualization and analysis methods need to be adapted to the specific needs of the particular application domain. For example, there are specialized systems for visualization of bio-chemical structures, shareholding structures and many more. Designing graph visualization systems with fast adaptability to various data types, analytical tasks and application-dependent analytical processes is still a challenge. Even within one application, often, the network to be analysed needs to be constructed from heterogeneous data sources, and the focus of interest (attributes of nodes and edges) varies dynamically. Designing such systems is obviously not trivial.

\subsection{Conceptual issues}

\subsubsection{Evaluation}

Evaluation of usability and user acceptability of the techniques including development of the evaluation methodologies is an important future challenge for the Visual Analytics research area [KMS*08, TC05, TC06, LK07]. Currently, there is a broad discussion in the Visual Analytics com- munity on the appropriate methodology for the evaluation of Visual Analytics and information visualization systems. This discussion applies also to the visual analysis of graphs. This challenge is expressed in the words of Plaisant et al. in the introduction to the special issue of Computer Graphics and Applications [PGS09] 'Assessing VA [Visual Analytics] technology's effectiveness is challenging because VA tools combine several disparate components, both low and high level, integrated in complex interactive systems used by analysts, emergency responders, and others. ... Traditional evaluation metrics such as task completion time, number of errors, or recall and precision are insufficient to quantify the utility of VA tools, and new research is needed to improve our VA evaluation methodology'. When concentrating on the evaluation of graph visualization techniques, several approaches have been proposed, ranging from quantitative to qualitative studies. Controlled experiments measuring accuracy and duration of user tasks have been used, for example, to compare tree visualization techniques [Kob04, AK07, ZK08]. An extension of these two main measures, the socalled cognitive load measure was used for evaluating general graph visualizations [HEH09]. Moreover, eye tracking can be employed for quantitative evaluation, for example for comparing graph layouts [Hua07, PSD09]. These controlled studies offer a quantitative comparison across techniques, however often suffer from the focus only on selected low level tasks. Note that the formulation of these tasks can influence the comparison result [ZK08]. A combination of quantitative and qualitative study has been performed for comparing graph layouts produced by both in a manual and in an algorithmic way [DLF*09]. The subjective user view has been used for ranking of best layouts. A qualitative view on the effectiveness of visual analytics techniques can be gained by use case studies conducted by domain experts (e.g. in [PS08, MGT*03]). This method offers insights into the usability of the systems in real world scenarios, however does not allow for standardized quantitative comparison of the techniques. The choice of appropriate evaluation method and its design is still discussed in the community.

\subsubsection{Taxonomies and benchmarks}

The field of visual graph analysis would profit from more elaborate taxonomies for tasks, interaction, visualization techniques, measures for quality, and benchmarks for comparing the new techniques. They would support both the design and development of visual analytic systems and their evaluation. Although several taxonomies and sample data sets exist, a more broader scope of theory and data aspects is needed owing to the large set of problems/tasks in visual analysis of graphs.

\section{Acknowledgments}

We thank Vyara Ivanova, Anna Mitkova, Melanie Görner and Robert Rehner for their helpful comments and 
suggestions. We are grateful to the anonymous reviewers for their constructive and useful comments. We thank all authors and copyright holders of the original figures for agreeing to their reproduction in this paper. This work was partially supported by the German Federal Ministry of Economics and Technology within the THESEUS project (http://www.theseus-programm.de/). It was also partially supported by the German Research Foundation (DFG) within the project Visual Feature Space Analysis as part of the Priority Program on Scalable Visual Analytics (SPP 1335).

\section{References}

[AAM07] Archambault D., Auber D., Munzner T.: Topolayout: multilevel graph layout by topological features. IEEE Transactions on Visualization and Computer Graphics 13, 2 (2007), 305-317.

[ACJM03] Auber D., Chiricota Y., Jourdan F., Melancon G.: Multiscale visualization of small world networks. In Proceedings of IEEE Symposium on Information Visualization (Washington, DC, USA, 2003), IEEE Press, pp. $75-81$.

[ADWM04] Adai A. T., Date S. V., Wieland S., Marcotte E. M.: LGL: creating a map of protein function with an algorithm for visualizing very large biological networks. Journal of Molecular Biology 340, 1 (June 2004), 179-190.

[AF07] Appert C., Fekete J.-D.: Naviguer dans des grands arbres avec controltree. In Proceedings of International Conference of the Association Francophone d'Interaction Homme-Machine (New York, NY, USA, 2007), ACM, New York, pp. 139-142.

[AH98] Andrews K., Heidegger H.: Information slices: visualising and exploring large hierarchies using cascading, semi-circular discs. In Late Breaking Hot Topic Paper, Proceedings of IEEE Symposium on Information Visualization (Washington, DC, USA, 1998), IEEE Press.

[AK07] AndRews K., KasanicKa J.: A comparative study of four hierarchy browsers using the hierarchical visualisation testing environment (HVTE). In Proceedings of International Conference Information Visualization (Washington, DC, USA, 2007), IEEE Press, pp. 81-86.

[AMA08] Archambault D., Munzner T., Auber D.: Grouseflocks: Steerable exploration of graph hierarchy space. IEEE Transactions on Visualization and Computer Graphics 14, 4 (July/August 2008), 900-913.

[AMA09] Archambault D., Munzner T., Auber D.: Tuggraph: path-preserving hierarchies for browsing proximity and paths in graphs. In Proceedings of IEEE Pacific Visu- alization Symposium (Beijing, China, 2009), IEEE Press, pp. 113-120.

[APP10] Archambault D., Purchase H. C., Pinaud B.: Difference map readability for dynamic graphs. In Proceedings of International Symposium on Graph Drawing (Konstanz, Germany, 2010), Lecture Notes in Computer Science (Springer Verlag, Berlin), pp. 50-61.

[Arc09] Archambault D.: Structural differences between two graphs through hierarchies. In Proceedings of Graphics Interface (Toronto, ON, Canada, 2009), Canadian Information Processing Society, pp. 87-94.

[AvH04] Abello J., van Ham F.: Matrix zoom: a visual interface to semi-external graphs. In Proceedings of IEEE Symposium on Information Visualization (Washington, DC, USA, 2004), IEEE, New York, pp. 183190.

[AWS92] Ahlberg C., Williamson C., Shneiderman B.: Dynamic queries for information exploration: an implementation and evaluation. In Proceedings of the SIGCHI Conference on Human Factors in Computing Systems (New York, NY, USA, 1992), ACM Press, pp. 619626.

[AWW09] Andrews K., Wohlfahrt M., Wurzinger G.: Visual graph comparison. In Proceedings of International Conference on Information Visualisation (Columbus, USA, 2009), IEEE Press, pp. 62-67.

[BBbl09] Bachmaier C., Brandenburg F. J., Brunner W., LovÁsz G.: Cyclic leveling of directed graphs. Lecture Notes in Computer Science 5417 (2009), Springer Verlag, Heidelberg, 348-359.

[BBD08] Burch M., Beck F., Dienl S.: Timeline trees: visualizing sequences of transactions in information hierarchies. In Proceedings of the Working Conference on Advanced Visual Interfaces (Napoli, Italy, 2008), ACM Press, New York, pp. 75-82.

[BBD09] Beck F., BuRCh M., DienL S.: Towards an aesthetic dimensions framework for dynamic graph visualisations. In Proceedings of the International Conference on Information Visualisation (Columbus, USA, 2009), IEEE Press, pp. 592-597.

[BBG*09] Blaas J., Botha C., Grundy E., Jones M., Laramee R., Post F.: Smooth graphs for visual exploration of higher-order state transitions. IEEE Transactions on Visualization and Computer Graphics 15, 6 (2009), 969-976.

[BC03] Brandes U., Corman S. R.: Visual unrolling of network evolution and the analysis of dynamic discourse. Information Visualization 2 (2003), 40-50. 
[BCD*10] Bezerianos A., Chevalier F., Dragicevic P., Elmqvist N., Fekete J.-D.: Graphdice: a system for exploring multivariate social networks. Computer Graphics Forum 29, 3 (2010), 863-872.

[BD08] Burch M., Dienl S.: Timeradartrees: visualizing dynamic compound digraphs. Computer Graphics Forum 27, 3 (2008), 823-830.

[BDF*10] Bezerianos A., Dragicevic P., Fekete J.-D., Bae J., Watson B.: GeneaQuilts: a system for exploring large genealogies. IEEE Transactions on Visualization and Computer Graphics 16, 6 (2010), 1073-1081.

[BDJ05] Brodlie K. W., Duke D. J., Joy K. I.: Arctrees: visualizing relations in hierarchical data. In Proceedings of the Joint Eurographics and IEEE TCVG Symposium on Visualization. P. Neumann, S. Schlechtweg and S. Carpendale (Eds.) (Leeds, UK, 2005), IEEE Press, pp. 53-60.

[BDL05] Balzer M., Deussen O., Lewerentz C.: Voronoi treemaps for the visualization of software metrics. In Proceedings of ACM Symposium on Software Visualization (Saint Louis, Missouri, USA, 2005), ACM, New York, pp. 165-172.

[BDL*10] Batagelu V., Didimo W., Liotta G., Palladino P., Patrignani M.: Visual analysis of large graphs using (x,y)-clustering and hybrid visualizations. In Proceedings of IEEE Pacific Visualization Symposium (Taipei, Taiwan, 2010), IEEE Press, pp. 209-216.

[Bed01] Bederson B. B.: Photomesa: a zoomable image browser using quantum treemaps and bubblemaps. In Proceedings of ACM Symposium on User Interface Software and Technology (Orlando, Florida, 2001), ACM, New York, pp. 71-80.

[BHvW99] Bruls M., Huizing K., van Wijk J.: Squarified treemaps. In Proceedings of the Joint Eurographics and IEEE TCVG Symposium on Visualization (Vienna, Austria, 1999), IEEE Press, pp. 33-42.

[BL09] Bertini E., Lalanne D.: Surveying the complementary role of automatic data analysis and visualization in knowledge discovery. In Proceedings of the ACM SIGKDD Workshop on Visual Analytics and Knowledge Discovery (Paris, France, 2009), ACM, New York, pp. 12-20.

[BMGK08] Barsky A., Munzner T., Gardy J., Kincaid R.: Cerebral: visualizing multiple experimental conditions on a graph with biological context. IEEE Transactions on Visualization and Computer Graphics 14, 6 (2008), $1253-1260$

[BMZ*06] Brennan S., Mueller K., Zelinsky G., Ramakrishnan I., Warren D., Kaufman A.: Toward a multi-analyst, collaborative framework for visual analytics. In Proceedings of IEEE Symposium on Visual Analytics Science and Technology (Baltimore, Maryland, USA, 2006), IEEE Press, pp. 129-136.

[BN01] Barlow T., Neville P.: A comparison of 2-D visualizations of hierarchies. In Proceedings of IEEE Symposium on Information Visualization (San Diego, CA, USA, 2001), IEEE Press, pp. 131-138.

[BRL09] Bertini E., Rigamonti M., Lalanne D.: Extended excentric labeling. Computer Graphics Forum 28 (2009), 927-934.

[BRSG07] Bennett C., Ryall J., Spalteholz L., Gooch A.: The aesthetics of graph visualization. In Proceedings of Computational Aesthetics in Graphics, Visualization, and Imaging (Banff, Alberta, Canada, 2007), Eurographics, Switzerland

[BSP*93] Bier E. A., Stone M. C., Pier K., Buxton W., Derose T. D.: Toolglass and Magic Lenses: the SeeThrough Interface. In Proceedings of the 20th Annual Conference on Computer Graphics and Interactive Techniques (New York, NY, USA, 1993), SIGGRAPH '93, ACM Press, New York, pp. 73-80.

[BSW02] Bederson B. B., Shneiderman B., Wattenberg M.: Ordered and quantum treemaps: making effective use of 2D space to display hierarchies. ACM Transactions on Graphics 21, 4 (2002), 833-854.

[CBTT95] Cohen R. F., Battista G. D., Tamassia R., Tollis I. G.: Dynamic graph drawings: trees, series-parallel digraphs, and planar st-digraphs. SIAM Journal of Computer 24 (1995), 970-1001.

[CC07] Collins C., Carpendale S.: VisLink: revealing relationships amongst visualizations. IEEE Transactions on Visualization and Computer Graphics 13, 6 (2007), 1192-1199.

[CGK*07] Chang R., Ghoniem M., Kosara R., Ribarsky W., Yang J., Suma E., Ziemkiewicz C., Kern D., Sudjianto A.: WireVis: visualization of categorical, time-varying data from financial transactions. In Proceedings of IEEE Symposium on Visual Analytics Science and Technology (Sacramento, CA, USA, 2007), IEEE Press, pp. 155162.

[CJM04] Chiricota Y., Jourdan F., Melancon G.: Metricbased network exploration and multiscale scatterplot. In IEEE Symposium on Information Visualization (Austin, Texas, 2004), IEEE Press, pp. 135-142.

[CMS99] Card S. C., Mackinlay J., Shneiderman B.: Readings in Information Visualization: Using Vision to Think. Morgan Kaufmann Publishers, 1999. 
[CN02] Card S. K., Nation D.: Degree-of-interest trees: a component of an attention-reactive user interface. In Proceedings of the Working Conference on Advanced Visual Interfaces (New York, NY, USA, 2002), ACM Press, New York, pp. 231-245.

[CR98] Chi E. H.-H., RiedL J. T.: An operator interaction framework for visualization systems. In proceeding of the IEEE Symposium on Information Visualization (Research Triangle Park, NC, USA, 1998), IEEE Press, pp. 63-70.

[CSP*06] Card S. K., Sun B., Pendleton B. A., Heer J., Bodnar J. W.: TimeTree: exploring time changing hierarchies. Proceeding of the Symposium on Visual Analytics Science and Technology (Baltimore, Maryland, 2006), IEEE Press, pp. 3-10.

[CZQ*08] Cui W., Zhou H., Qu H., Wong P. C., Li X.: Geometry-based edge clustering for graph visualization. IEEE Transactions on Visualization and Computer Graphics 14, 6 (2008), 1277-1284.

[DBETT99] Di Battista G., Eades P., Tamassia R., Tollis I. G.: Graph Drawing: Algorithms for the Visualization of Graphs. Prentice-Hall, 1999.

[DGK01] Diehl S., Goerg C., Kerren A.: Preserving the mental map using foresighted layout. In Proceedings of Joint Eurographics, IEEE TCVG Symposium on Visualization (Ascona, Switzerland, 2001), IEEE Press, pp. $175-184$.

[DHKS05] Dayal U., Hao M., Keim D., Schreck T.: Importance driven visualization layouts for large time-series data. In Proceedings of IEEE Symposium on Information Visualization (2005), IEEE Press, pp. 203-210.

[Die05] Diestel R.: Graph Theory. Springer-Verlag, Heidelberg, 2005.

[DK05] Dwyer T., Koren Y.: DIG-COLA: directed graph layout through constrained energy minimization. In Proceedings of IEEE Symposium on Information Visualization (Minneapolis, MN, USA, 2005), IEEE Press, p. 9.

[DLF*09] Dwyer T., LeE B., Fisher D., QuinN K. I., Isenberg P., Robertson G., North C.: A comparison of user-generated and automatic graph layouts. IEEE Transactions on Visualization and Computer Graphics 15, 6 (2009), IEEE Press, pp. 961-968.

[dMB05] de Nooy W., Mrvar A., Batagelu V.: Exploratory Social Network Analysis with Pajek. Cambridge University Press, Cambridge, USA, 2005.

[DMS*08] Dwyer T., Marriott K., Schreiber F., Stuckey P., Woodward M., Wybrow M.: Exploration of networks using overview+detail with constraint-based cooperative layout. IEEE Transactions on Visualization and Computer Graphics 14, 6 (2008), 1293-1300.

[DMW09a] Dwyer T., Marriott K., Wybrow M.: Dunnart: a constraint-based network diagram authoring tool. Graph Drawing 5417 (2009), 420-431.

[DMW09b] Dwyer T., Marriott K., Wybrow M.: Topology preserving constrained graph layout. In Proceedings of the Revised Papers from International Symposium on Graph Drawing (Chicago, USA, 2009), Lecture Notes in Computer Science, Springer Verlag, Heidelberg, pp. 230-241.

[Dog02] Dogrusoz U.: Two-dimensional packing algorithms for layout of disconnected graphs. Information Sciencies 143, 1-4 (2002), 147-158.

[DPS02] Díaz J., Petit J., Serna M.: A survey of graph layout problems. ACM Computing Surveys 34, 3 (2002), 313-356.

[Ead84] Eades P.: A heuristic for graph drawing. Congressus Numerantium 42 (1984), 149-160.

[EDF10] Elmqvist N., Dragicevic P., Fekete J.-D.: Color lens: adaptive color scale optimization for visual exploration. IEEE Transactions on Visualization and Computer Graphics 99 (2010), RapidPosts, IEEE Press.

[EDG*08] Elmqvist N., Do T.-N., Goodell H., Henry N., FEKETE J.-D.: Zame: interactive large-scale graph visualization. In Proceedings of IEEE Pacific Visualization Symposium (Kyoto, Japan, 2008), IEEE Press, pp. 215-222.

[EF09] Elmqvist N., FeKETE J.-D.: Hierarchical aggregation for information visualization: overview, techniques, and design guidelines. IEEE Transactions on Visualization and Computer Graphics 16, 3 (2009), 439-454.

[EHK*03] Erten C., Harding P. J., Kobourov S. G., WAMPLeR K., YEe G.: Graphael: graph animations with evolving layouts. In Proceedings of the 11th Symposium on Graph Drawing (Perugia, Italy, 2003), IEEE Press, pp. 98-110.

[ElMS91] Eades P., Lai W., Misue K., Sugiyama K.: Preserving the Mental Map of a Diagram. Research Report IIAS-RR-91-16E, International Institute for Advanced Study of Social Information Science, August 1991.

[ERHF09] Elmovist N., Riche Y., Henry N., Fekete J.D.: Mélange: Space folding for visual exploration. IEEE Transactions on Visualization and Computer Graphics 99 , 1 (2009)

[FDK02] Freivalds K., Dogrusöz U., KiKusts P.: Disconnected graph layout and the polyomino packing approach. In Revised Papers from the 9th Int. Symposium 
on Graph Drawing (London, UK, 2002), Springer-Verlag, pp. 378-391.

[FHK*09] Fung D. C. Y., Hong S.-H., Koschutzki D., Schreiber F., Xu K.: Visual analysis of overlapping biological networks. In Proceedings of the 2009 13th International Conference Information Visualisation (Washington, DC, USA, 2009), IEEE Computer Society, pp. 337-342.

[FLM95] Frick A., Ludwig A., Mehldau H.: A fast adaptive layout algorithm for undirected graphs. In Proceedings of the DIMACS International Workshop on Graph Drawing (1995), pp. 388-403.

[FP99] Fekete J.-D., Plaisant C.: Excentric labeling: dynamic neighborhood labeling for data visualization. In Proceedings of the SIGCHI conference on Human factors in computing systems: the CHI is the limit (New York, NY, USA, 1999), CHI '99, ACM, pp. 512-519.

[FPSG10] Freire M., Plaisant C., Shneiderman B., GolBECK J.: ManyNets: an interface for multiple network analysis and visualization. In Proceedings of international conference on Human factors in computing systems (New York, NY, USA, 2010), ACM, pp. 213-222.

[FR91] Fruchterman T. M. J., Reingold E. M.: Graph drawing by force-directed placement. Software: Practice and Experience 21, 11 (1991), 1129-1164.

[Fre79] Freeman L. C.: Centrality in social networks. Social Networks 1, 3 (1979), 215-239.

[FT04] Frishman Y., TAL A.: Dynamic drawing of clustered graphs. In Proceedings of the IEEE Symposium on Information Visualization (Austin, TX, USA, 2004), IEEE Press, pp. 191-198.

[FT07] Frishman Y., Tal A.: Multi-level graph layout on the GPU. IEEE Transactions on Visualization and Computer Graphics 13, 6 (2007), 1310-1319.

[FT08] Frishman Y., Tal A.: Online dynamic graph drawing. IEEE Transactions on Visualization and Computer Graphics 14, 4 (2008), 727-740.

[Fur86] Furnas G. W.: Generalized fisheye views. SIGCHI Bulletin 17, 4 (1986), 16-23.

[FWD*03] Fekete J.-D., Wang D., Dang N., Aris A., Plaisant C.: Overlaying graph links on treemaps. In Proceedings of IEEE Information Visualization Symposium Posters Compendium (Seattle, USA, 2003), IEEE Press.

[GBD09] Greilich M., Burch M., Diehl S.: Visualizing the evolution of compound digraphs with TimeArcTrees. Computer Graphics Forum 28, 3 (2009), 975982.
[GBPD04] Görg C., Birke P., Pohl M., Diehl S.: Dynamic graph drawing of sequences of orthogonal and hierarchical graphs. In Graph Drawing (2004), Pach J. (Ed.), vol. 3383 of Lecture Notes in Computer Science, Springer, Heidelberg, pp. 228-238.

[GDLP09] Giacomo E., Didimo W., Liotta G., Palladino P.: Visual analysis of one-to-many matched graphs. In Proceedings of the Revised Papers from International Symposium on GraphDrawing (Konstanz, Germany, 2009), Springer Verlag, Heidelberg, pp. 133-144.

[GF01] Ghoniem M., Fekete J.-D.: Animating treemaps. In Proceedings of 18th HCIL Symposium-Workshop on Treemap Implementations and Applications (Maryland, USA, 2001), University of Maryland, College Park, USA.

[GFC04] Ghoniem M., Fekete J.-D., Castagliola P.: A comparison of the readability of graphs using node-link and matrix-based representations. In Proceedings of IEEE Symposium on Information Visualization (Austin, TX, USA, 2004), IEEE Press, pp. 17-24.

[GH09] Gansner E. R., Hu Y: Efficient node overlap removal using a proximity stress model. Graph Drawing 5417 (2009), 206-217.

[GHGH09] Godiyal A., Hoberock J., Garland M., Hart J. C.: Rapid multipole graph drawing on the gpu. In Graph Drawing,Tollis I. G. and Patrignani M. (Eds.). SpringerVerlag, Berlin, Heidelberg, 2009, pp. 90-101.

[GHK10] Gansner E., Hu Y., Kobourov S.: GMap: visualizing graphs and clusters as maps. In Proceedings of IEEE Pacific Visualization Symposium (Taipei, Taiwan, 2010), IEEE Press, pp. 210-208.

[GK01] Gajer P., Kobourov S. G.: GRIP: Graph drawing with intelligent placement. In Proceedings of International Symposium on Graph Drawing (Vienna, Austria, 2001), Springer Verlag, Heidelberg, pp. 222-228.

[GKNV93] Gansner E. R., Koutsofios E., North S. C., Vo K.-P.: A technique for drawing directed graphs. IEEE Transactions on Software Engineering 19 (1993), 214-230.

[GKS07] Goehlsdorf D., Kaufmann M., Siebenhaller M.: Placing connected components of disconnected graphs. In Proceedings of Asia-Pacific Symposium on Information Visualisation (Kyoto, Japan, 2007), IEEE Press, pp. 101-108.

[GOB*10] Gretarsson B., O'Donovan J., Bostandjev S., HALL C., Höllerer T.: SmallWorlds: Visualizing social recommendations. Computer Graphics Forum 29, 3 (2010), 833-842. 
[GS05] Griethe H., Schumann H.: Visualizing uncertainty for improved decision making. In Proceedings of International Conference on Perspectives in Business Informatics Research (Karlstad, Sweden, 2005), Skovde, Sweden.

[GS06] Griethe H., Schumann H.: The visualization of uncertain data: methods and problems. In Proceedings of Simulation and Visualization (Trier, Germany, 2006), SCS Publishing House, GB, pp. 143-156.

[GZ08] Goтz D., Zнои M.: Characterizing users' visual analytic activity for insight provenance. In Proceedings of IEEE Symposium on Visual Analytics Science and Technology (Columbus, USA, 2008), IEEE Press, pp. 123-130.

[HB05] HeER J., Boyd D.: Vizster: visualizing online social networks. In Proceedings of the IEEE Symposium on Information Visualization (Minneapolis, MN, USA, 2005), IEEE Press, pp. 32-39.

[HC04] Heer J., Card S. K.: Doitrees revisited: scalable, space-constrained visualization of hierarchical data. In Proceedings of the Working Conference on Advanced Visual Interfaces (New York, NY, USA, 2004), ACM Press, New York, pp. 421-424.

[HD10] Henry Riche N., Dwyer T.: Untangling Euler diagrams. IEEE Transactions on Visualization and Computer Graphics 16, 6 (2010), 1090-1099.

[HeH09] Huang W., Eades P., Hong S.-H.: Measuring effectiveness of graph visualizations: a cognitive load perspective. Information Visualization 8 (2009), 139 152.

[HF06] Henry N., Fekete J.-D.: MatrixExplorer: a dualrepresentation system to explore social networks. IEEE Transactions on Visualization and Computer Graphics 12, 5 (2006), 677-684.

[HF07] Henry N., Fekete J.-D.: Matlink: enhanced matrix visualization for analyzing social networks. In Proceedings of the International Conference on HumanComputer-Interaction (Rio de Janeiro, Brasil, 2007), Springer Verlag, Berlin, pp. 288-302.

[HFM07] Henry N., Fekete J.-D., McGuffin M.: NodeTrix: a hybrid visualization of social networks. IEEE Transactions on Visualization and Computer Graphics 13, 6 (2007), 1302-1309.

[HJ05] Hachul S., Jünge M.: Drawing large graphs with a potential-field-based multilevel algorithm. Lecture notes in Computer Science 4372 (2005), 285-295.

[HJ07] Hachul S., JüNGER M.: Large-graph layout algorithms at work: an experimental study. Journal of Graph Algorithms and Applications 11, 2 (2007), 234-369.
[HK02] Harel D., Koren Y.: Graph drawing by highdimensional embedding. In Proceedings of the Revised Papers from International Symposium on Graph Drawing (Irvine, CA, USA, 2002), Springer Verlag, Berlin, pp. 207-219.

[HMM00] Herman I., Melancon G., Marshall M. S.: Graph visualization and navigation in information visualization: a survey. IEEE Transactions on Visualization and Computer Graphics 6, 1 (2000), 24-43.

[HMSA08] Heer J., Mackinlay J., Stolte C., Agrawala M.: Graphical histories for visualization: supporting analysis, communication, and evaluation. IEEE Transactions on Visualization and Computer Graphics 14, 6 (2008), 1189-1196.

[Hol06] HoLTEN D.: Hierarchical edge bundles: visualization of adjacency relations in hierarchical data. IEEE Transactions on Visualization and Computer Graphics 12, 5 (2006), 741-748.

[Hua07] Huang W.: Using eye tracking to investigate graph layout effects. In Proceedings of Asia-Pacific Symposium on Visualization (Kyoto, Japan, 2007), IEEE Press, pp. 97-100.

[HvW08] Holten D., van WiJk J. J.: Visual comparison of hierarchically organized data. Computer Graphics Forum 27, 3 (2008), 759-766.

[HvW09] Holten D., van WiJk J. J.: A user study on visualizing directed edges in graphs. In Proceedings of International Conference on Human Factors in Computing Systems (Boston, MA, USA, 2009), ACM, New York, pp. 2299-2308.

[IAG*09] Imamichi T., Arahori Y., Gim J., Hong S.-H. NAGAMOCHI H.: Removing node overlaps using multisphere scheme. In Proceedings of the Graph Drawing (Konstanz, Germany, 2009), pp. 296-301.

[ITC08] Isenberg P., Tang A., Carpendale S.: An exploratory study of visual information analysis. In Proceedings of the Conference on Human Factors in Computing Systems (2008), ACM Press, pp. 1217-1226.

[JHGH08] Jia Y., Hoberock J., Garland M., Hart J.: On the visualization of social and other scale-free networks. IEEE Transactions on Visualization and Computer Graphics 14, 6 (2008), 1285-1292.

[JS10] Jürgensmann S., Schulz H.-J.: Poster: a visual survey of tree visualization. In Proceedings of IEEE Information Visualization (Salt Lake City, USA, 2010), IEEE Press

[KAF*08] Keim D., Andrienko G., Fekete J.-D., Görg C., Kohlhammer J., Melancon G.: Information Visualization, 
vol. 4950 of Lecture Notes in Computer Science. Springer, 2008, ch. Visual Analytics: Definition, Process, and Challenges, pp. 154-175.

[KCH02] Koren Y., Carmel L., Harel D.: ACE: A fast multiscale eigenvectors computation for drawing huge graphs. In Proceedings of the IEEE Symposium on Information Visualization (Boston, MA, USA, 2002), IEEE Press, p. 137.

[Kee06] KeEL P.: Collaborative visual analytics: inferring from the spatial organization and collaborative use of information. In Proceedings of IEEE Symposium on Visual Analytics Science and Technology (Baltimore, USA, 2006), IEEE Press, pp. 137-144.

[KG06] Kumar G., Garland M.: Visual exploration of complex time-varying graphs. IEEE Transactions on Visualization and Computer Graphics 12, 5 (2006), 805-812.

[KK89] Kamada T., KawaI S.: An algorithm for drawing general undirected graphs. Information Processing Letters 31, 1 (1989), Elsevier, 7-15.

[KKS09] Kaufmann M., Kreveld M., Speckmann B.: Subdivision drawings of hypergraphs. In Proceedings of the Revised Papers from International Symposium on Graph Drawing (2009), pp. 396-407.

[KMS*08] Keim D. A., Mansmann F., Schneidewind J., ThOmAs J., ZiEgler H.: Visual analytics: scope and challenges, visual data mining: theory, techniques and tools for visual analytics. Lecture Notes in Computer Science (LNCS) 4404 (2008), 76-90.

[Kob04] KobSa A.: User experiments with tree visualization systems. In Proceedings of the IEEE Symposium on Information Visualization (Austin, USA, 2004), IEEE Press, pp. 9-16.

[Koh01] Kohonen T.: Self-Organizing Maps, 3rd ed. Springer, Berlin, 2001

[Kre09] Krempel L.: Network visualization. In Handbook of Social Network Analysis, J. Scott and P. J. Carrington (Eds.). Sage Publishing, 2009, ch. 37.

[KSS06] Klukas C., Schreiber F., Schwöbbermeyer H.: Coordinated perspectives and enhanced force-directed layout for the analysis of network motifs. In Proceedings of AsiaPacific Symposium on Information Visualisation (Tokyo, Japan, 2006), IEEE Press, pp. 39-48.

[LBA10] Lambert A., Bourqui R., Auber D.: Winding roads: routing edges into bundles. Computer Graphics Forum 29, 3 (2010), 853-862.

[LF06] Leskovec J., Faloutsos C.: Sampling from large graphs. In Proceedings of ACM SIGKDD International
Conference on Knowledge Discovery and Data Mining (Philadelphia, USA, 2006), ACM, New York, pp. 631-636.

[LK07] Laramee R. S., Kosara R.: Challenges and unsolved problems. Lecture Notes in Computer Science 4417 (2007), 231-254.

[LPS*06] Lee B., Plaisant C., Sims Parr C., Fekete J.D., Henry N.: Task taxonomy for graph visualization. In Proceedings of AVI Workshop on BEyond time and Errors (Venezia, Italy, 2006), ACM, New York, pp. 1-5.

[LrCP07] Lee B., Robertson G. G., Czerwinski M., Parr C. S.: CandidTree: Visualizing structural uncertainty in similar hierarchies. Information Visualization 6 (December 2007), 233-246.

[LRP95] Lamping J., Rao R., Pirolli P.: A focus+context technique based on hyperbolic geometry for visualizing large hierarchies. In Proceedings of the SIGCHI Conference on Human Factors in Computing Systems (Denver, Colorado, USA, 1995), ACM Press/Addison-Wesley Publishing Co., New York, pp. 401-408.

[MCH*09] Moscovich T., Chevalier F., Henry N., Pietriga E., FEKETE J.-D.: Topology-aware navigation in large networks. In Proceedings of International Conference on $\mathrm{Hu}$ man Factors in Computing Systems (Boston, USA, 2009), ACM, New York, pp. 2319-2328.

[Mel06] Melancon G.: Just how dense are dense graphs in the real world: a methodological note. In Proceedings of AVI Workshop on BEyond Time and Errors: Novel Evaluation Methods for Information Visualization (2006), ACM Press, New York, pp. 1-7.

[Mey98] Meyer B.: Competitive learning of network diagram layout. In Proceedings of IEEE Symposium on Visual Languages (Halifax, NS, Canada, Canada, 1998), IEEE Press, pp. 56-63.

[MGL06] Mueller C., Gregor D., Lumsdaine A.: Distributed force-directed graph layout and visualization. In Proceedings of the Eurographics Symposium on Parallel Graphics and Visualization (Lisbon, Portugal, 2006), IEEE Press.

[MGT*03] Munzner T., Guimbretière F., Tasiran S., Zhang L., Zно Y.: TreeJuxtaposer: scalable tree comparison using focus + context with guaranteed visibility. In Proceedings of ACM SIGGRAPH (San Diego, USA, 2003), ACM, New York, pp. 453-462.

[MJ09] McGuffin M. J., JuRisica I.: Interaction techniques for selecting and manipulating subgraphs in network visualizations. IEEE Transactions on Visualization and Computer Graphics 15 (2009), 937-944. 
[MJW*09] Ma'ayan A., Jenkins S., Webb R., Berger S., Purushothaman S., Husn N. A., Posner J., Flores T., IYENGAR R.: SNAVI: Desktop application for analysis and visualization of large-scale signaling networks. BMC Systems Biology 3, 1 (2009), 10-20.

[MM08] Muelder C., Ma K.-L.: Rapid graph layout using space filling curves. IEEE Transactions on Visualization and Computer Graphics, 14, 6 (2008), 1301-1308.

[MML07] Mueller C., Martin B., Lumsdaine A.: A comparison of vertex ordering algorithms for large graph visualization. Proceedings of International AsiaPacific Symposium on Visualization (Kyoto, Japan, 2007), IEEE Press, pp. 141-148.

[MMO05] Mcpherson J., Ma K.-L., Ogawa M.: Discovering parametric clusters in social small-world graphs. In Proceedings of ACM Symposium on Applied Computing (Santa Fe, USA, 2005), ACM, New York, pp. 12311238.

[Mun97] MunzNER T.: H3: laying out large directed graphs in 3D hyperbolic space. In Proceedings of IEEE Symposium on Information Visualization (Phoenix, AZ, USA, 1997), pp. 2-10, 114.

[NCA06] Neumann P., Carpendale M. S. T., Agarawala A.: Phyllotrees: phyllotactic patterns for tree layout. In Proceedings of the Joint Eurographics and IEEE TCVG Symposium on Visualization (Lisbon, Portugal, 2006), Eurographics, pp. 59-66.

[Noa03] NoAck A.: Energy models for drawing clustered small-world graphs. In Proceedings of International Symposium on Graph Drawing (Perugia, Italy, 2003), Springer Verlag, Berlin, pp. 425-436.

[Nor96] NorTh S. C.: Incremental layout in DynaDAG. In Proceedings of the Symposium on Graph Drawing (Passau, Germany, 1996), Springer-Verlag, Berlin, pp. 409-418.

[OCNF09] Otjacques B., Cornil M., Noirhomme M., Feltz F.: CGD - a new algorithm to optimize space occupation in ellimaps. In Proceedings of IFIP TC 13 International Conference on Human-Computer Interaction (San Diego, USA, 2009), Springer Verlag, Berlin, pp. 805-818.

[OPPROG09] Ovalle-Perandones M. A., PerianesRodriguez A., Olmeda-Gomez C.: Hubs and authorities in a Spanish co-authorship network. In Proceedings of International Conference Information Visualisation (Atlantic City, USA, 2009), IEEE Press, pp. 514518.

[PD08] PoHL M., Dienl S.: What dynamic network metrics can tell us about developer roles. In Proceedings of the International Workshop on Cooperative and Human Aspects of Software Engineering (New York, NY, USA, 2008), ACM Press, pp. 81-84.

[PGB02] Plaisant C., Grosjean J., Bederson B. B.: Spacetree: supporting exploration in large node link tree, design evolution and empirical evaluation. In Proceedings of the IEEE Symposium on Information Visualization (Boston, USA, 2002), IEEE Press, p. 57.

[PGS09] Plaisant C., Grinstein G., Scholtz J.: Visualanalytics evaluation. IEEE Computer Graphics and Applications 29, 3 (2009), 16-17.

[PRB08] Pohl M., Reitz F., Birke P.: As time goes by: integrated visualization and analysis of dynamic networks. In Proceedings of the Working Conference on Advanced Visual Interfaces (Napoli, Italy, 2008), ACM, New York, pp. 372-375.

[PS06] Perer A., Shneiderman B.: Balancing systematic and flexible exploration of social networks. IEEE Transactions on Visualization and Computer Graphics 12 (2006), 693-700.

[PS08] Perer A., Shneiderman B.: Integrating statistics and visualization: case studies of gaining clarity during exploratory data analysis. In Proceeding of SIGCHI Conference on Human Factors in Computing Systems (Florence, Italy, 2008), IEEE Press, pp. 265-274.

[PSD09] Pohl M., Schmitt M., Diehl S.: Comparing readability of graph layouts using eyetracking and taskoriented analysis. In Proceedings of Computer Graphics International (Victoria, British Columbia, Canada, 2009), Victoria, Canada.

[Pur97] Purchase H. C.: Which aesthetic has the greatest effect on human understanding\&quest. In Proceedings of International Symposium on Graph Drawing (Rome, Italy, 1997), Springer Verlag, Berlin, pp. 248-261.

[PWL97] Pang A., Wittenbrink C., Lodha. S.: Approaches to uncertainty visualization. The Visual Computer 13, 8 (1997), 370-390.

[RMC91] Robertson G. G., Mackinlay J. D., Card S. K.: Cone Trees: animated 3D visualizations of hierarchical information. In Proceedings of the SIGCHI Conference on Human Factors in Computing Systems (New Orleans, USA, 1991), ACM, New York, pp. 189194.

[RPD09] Reitz F., Pohl M., Dienl S.: Focused animation of dynamic compound graphs. In Proceedings of International Conference Information Visualisation (Atlantic City, USA, 2009), IEEE Press, pp. 679684. 
[SA06] Shneiderman B., Aris A.: Network visualization by semantic substrates. IEEE Transactions on Visualization and Computer Graphics, 12, 5 (2006), 733-740.

[SB92] Sarkar M., Brown M. H.: Graphical fisheye views of graphs. In Proceedings of the SIGCHI conference on Human factors in computing systems (1992), Springer Verlag, Berlin, pp. 83-91.

[SCGM00] Stasko J., Catrambone R., Guzdial M., MCDONALD K.: An evaluation of space-filling information visualizations for depicting hierarchical structures. International Journal of Human-Computer Studies 53, 5 (2000), 663-694.

[Sch08] Schwöвbermeyer H.: Analysis of Biological Networks. Wiley Series on Bioinformatics, Computational Techniques and Engineering. Wiley, 2008, ch. 5, pp. $85-112$.

[SFL10] Sud A., Fisher D., LeE H.-P.: Fast dynamic voronoi treemaps. In Proceedings of International Symposium on Voronoi Diagrams in Science and Engineering (2010), pp. 85-94.

[Shn92] Shneiderman B.: Tree visualization with tree-maps: 2-D space-filling approach. ACM Transactions on Graphics 11, 1 (1992), 92-99.

[SKM06] Schreck T., Keim D. A., Mansmann S.: Regular treemap layouts for visual analysis of hierarchical data. In Proceedings of Spring Conference on Computer Graphics (2006), Comenius University, Bratislava, pp. 184-191.

[SLN05] Saraiya P., Lee P., North C.: Visualization of graphs with associated timeseries data. In Proceedings of IEEE Symposium on Information Visualization (2005), IEEE Press, pp. 225-232.

[SS05] Schreiber F., Schwöbbermeyer H.: MAVisto: a tool for the exploration of network motifs. Bioinformatics 21, 17 (2005), 3572-3574.

[SSH09] Schulz H.-J., Schumann H., HadlaK S.: Pointbased tree representation - a new approach for large hierarchies. In Proceedings of IEEE Pacific Visualization Symposium (2009), IEEE Press, pp. 81-88.

[STT81] SugiYama K., Tagawa S., Toda M.: Methods for visual understanding of hierarchical system structures. Systems, Man and Cybernetics, IEEE Transactions on 11,2 (1981), 109-125.

[SZ00] Stasko J., Zhang E.: Focus+context display and navigation techniques for enhancing radial, space-filling hierarchy visualizations. In Proceedings of the IEEE Symposium on Information Vizualization (Salt Lake City, USA, 2000), p. 57.
[SZG*96] Schaffer D., Zuo Z., Greenberg S., Bartram L., Dill J., Dubs S., Roseman M.: Navigating hierarchically clustered networks through fisheye and full-zoom methods. ACM TOCHI 3, 2 (1996), 162 188.

[TAS09] Tominski C., Abello J., Schumann H.: CGVan interactive graph visualization system. Computers \& Graphics 33, 6 (2009), 660-678.

[TAvHS06] Tominski C., Abello J., van Ham F., Schumann H.: Fisheye tree views and lenses for graph visualization. In Proceedings of IEEE Information Visualization (Baltimore, USA, 2006), IEEE Press, pp. 17-24.

[TC05] Thомаs J. J., Соок K. A. (Eds.): Illuminating the Path: The Research and Development Agenda for Visual Analytics. National Visualization and Analytics Center, IEEE Press, 2005.

[TC06] Tномаs J., Соок K.: A visual analytics agenda. Computer Graphics and Applications, IEEE 26, 1 (2006) 10-13.

[TE10] Telea A., ERsoy O.: Image-based edge bundles: Simplified visualization of large graphs. Computer Graphics Forum 29, 3 (2010), 843-852.

[THM*05] Thomson J., Hetzler B., Maceachren A., Gahegan M., Pavel M.: A typology for visualizing uncertainty. In Proceedings of SPIE Conference on Visualization and Data Analysis 2005 (San Jose, CA, USA 2005), vol. 5669, SPIE, Bellingham, WA, USA, pp. 146157.

[TK08] TeKuŠová T., Kohlhammer J.: Visual analysis and exploration of complex corporate shareholder networks. In Proceedings of the IS\&T/SPIE Visualization and Data Analysis (San Jose, CA, USA, 2008), vol. 6809, SPIE, Bellingham, WA, USA, p. 68090F.

[TS99] Toyoda M., Shibayama E.: Hyper mochi sheet: a predictive focusing interface for navigating and editing nested networks through a multi-focus distortion-oriented view. In Proceedings of the SIGCHI Conference on Human Factors in Computing Systems (Pittsburgh, PA, USA, 1999), ACM, New York, pp. 504-511.

[TS07] Tu Y., SHEN H.-W.: Visualizing changes of hierarchical data using treemaps. IEEE Transactions on Visualization and Computer Graphics 13, 6 (2007), 12861293.

[TS08a] Tekušová T., Schreck T.: Visualizing timedependent data in multivariate hierarchic plots - design and evaluation of an economic application. In Proceedings of International Conference on Information Visualisation (Columbus, USA, 2008), IEEE Press, pp. 143-150. 
[TS08b] Tu Y., SHEN H.-W.: Balloon focus: a seamless multifocus+context method for treemaps. IEEE Transactions on Visualization and Computer Graphics, 14, 6 (2008), 1157-1164.

[vH03] van HAM F.: Using multilevel call matrices in large software projects. In Proceedings of the IEEE Symposium on Information Visualization (Seattle, USA, 2003), IEEE Press, pp. 227-232.

[vHP09] van Ham F., Perer A.: Search, show context, expand on demand: supporting large graph exploration with degree-of-interest. IEEE Transactions on Visualization and Computer Graphics 15, 6 (2009), 953-960.

[vHR08] van Ham F., Rogowitz B.: Perceptual organization in user-generated graph layouts. IEEE Transactions on Visualization and Computer Graphics, 14, 6 (2008), 1333-1339.

[vHSD09] van Ham F., Schulz H.-J., Dimicco J. M.: Honeycomb: Visual analysis of large scale social networks. In Proceedings of IFIP TC 13 International Conference on Human-Computer Interaction: Part II (San Diego, USA, 2009), Springer Verlag, Heidelberg, pp. 429-442.

[vHvW02] van Ham F., van WiJk J.: Beamtrees: compact visualization of large hierarchies. In Proceedings of the IEEE Symposium on Information Visualization (Boston, USA, 2002), IEEE Press, pp. 93-100.

[vHW08] van Ham F., Wattenberg M.: Centrality based visualization of small world graphs. Computer Graphics Forum 27, 3 (2008), 975-982.

[vLdL03] van Liere R., De Leeuw W.: Graphsplatting: Visualizing graphs as continuous fields. IEEE Transactions on Visualization and Computer Graphics 9 (2003), 206212.

[vLGRS09] von LANDESBerger T., GÖrner M., Rehner R., SCHRECK T.: A system for interactive visual analysis of large graphs using motifs in graph editing and aggregation. In Proceedings of Vision Modeling Visualization Workshop (Atlantic City, USA, 2009), TU Braunschweig, Germany, pp. 331-339.

[vLGS09] von Landesberger T., Görner M., Schreck T.: Visual analysis of graphs with multiple connected components. In Proceedings of IEEE Symposium on Visual Analytics Science and Technology (Braunschweig, Germany, 2009), IEEE Press, pp. 155-162.

[VMCJ10] Viau C., McGuffin M. J., Chiricota Y., Jurisica I.: The FlowVizMenu and parallel scatterplot matrix: hybrid multidimensional visualizations for network exploration. IEEE Transactions on Visualization and Computer Graphics 16 (2010), 1100-1108.
[vWvdW99] van WiJK J., van de Wetering H.: Cushion treemaps: visualization of hierarchical information. In Proceedings of IEEE Symposium on Information Visualization (San Francisco, USA, 1999), IEEE Press, pp. 73-78.

[War00] WARE C.: Information Visualization: Perception for Design. Morgan Kaufmann, 2000.

[Wat06] WatTEnberg M.: Visual exploration of multivariate graphs. In Proceedings of the SIGCHI Conference on Human Factors in Computing Systems (Montreal, Canada, 2006), ACM, New York, pp. 811-819.

[WBS*08] Watson B., Brink D., Stallman M., Devajaran D., RhyNe T.-M., PATEL H.: Visualizing very Large Layered Graphs with Quilts. Tech. Rep. TR-2008-10, NC State University, 2008.

[WCG03] Wong N., Carpendale S., Greenberg S.: Edgelens: an interactive method for managing edge congestion in graphs. In Proceedings of IEEE Symposium on Information Visualization (Seattle, USA, 2003), IEEE Press, pp. 51--58.

[WD08] Wood J., Dykes J.: Spatially ordered treemaps. IEEE Transactions on Visualization and Computer Graphics, 14, 6 (2008), 1348-1355.

[WMC*09] Wong P. C., Mackey P., Cook K., Rohrer R., Foote H., Whiting M.: A multi-level middle-out crosszooming approach for large graph analytics. In Proceedings of IEEE Symposium on Visual Analytics Science and Technology (Atlantic City, USA, 2009), IEEE Press, pp. 147-154.

[WS03] White S., Smyth P.: Algorithms for estimating relative importance in networks. In Proceedings of ACM SIGKDD International Conference on Knowledge Discovery and Data Mining (NY, USA, 2003), ACM Press, pp. 266-275.

[YKSJ07] Y. J. S., Kang Y. A., Stasko J., Jacko J.: Toward a deeper understanding of the role of interaction in information visualization. IEEE Transactions on Visualization and Computer Graphics 13, 6 (2007), 1224 1231

[ZK08] Ziemkiewicz C., Kosara R.: The shaping of information by visual metaphors. IEEE Transactions on Visualization and Computer Graphics 14, 6 (2008), 12691276

[ZMC05] Zhao S., Mcguffin M., Chignell M.: Elastic hierarchies: combining treemaps and node-link diagrams. In Proceedings of IEEE Symposium on Information Visualization (Minneapolis, MN, USA, 2005), IEEE Press, pp. $57-64$. 